\title{
Moderate Deviation Asymptotics for Variable-Length Codes with Feedback
}

\author{
Lan V. Truong and Vincent Y. F. Tan
}

\begin{abstract}
We consider data transmission across discrete memoryless channels (DMCs) using variable-length codes with feedback. We consider the family of such codes whose rates are $\rho_{N}$ below the channel capacity $C$, where $\rho_{N}$ is a positive sequence that tends to zero slower than the reciprocal of the square root of the expectation of the (random) blocklength $N$. This is known as the moderate deviations regime and we establish the optimal moderate deviations constant. We show that in this scenario, the error probability decays sub-exponentially with speed $\exp \left(-(B / C) N \rho_{N}\right)$, where $B$ is the maximum relative entropy between output distributions of the DMC.
\end{abstract}

\section{Index Terms}

Variable-length codes, Feedback, Error exponent, Moderate deviations constant, Discrete memoryless channel

\section{INTRODUCTION}

Shannon showed [1] that feedback does not increase the capacity of memoryless channels. However, feedback has many practical advantages in various communication settings, including simplifying coding schemes [2], [3] and dramatically minimizing the error probability at finite blocklengths [4], [5]. This paper focuses on the simplest channel model—the discrete memoryless channel (DMC). We consider the scenario in which the length of the code is a random variable (in particular, a stopping time of the filtration generated by the sequence of channel outputs) and full feedback is available at the encoder. Different from previous works which consider the error exponents (large deviations) regime [4] and the fixed-error (secondorder) regime [5], we analyze the performance of codes whose transmission rates are $\rho_{N}$ below the channel capacity $C$, where $\rho_{N}$ is a positive sequence that tends to zero slower than the reciprocal of the square root of the expectation of the (random) blocklength $N$. This is known as the moderate deviations regime [6], [7]. We derive a tight result for the moderate deviations constant; this is defined precisely in Section II.

\section{A. Related Works}

Burnashev, in a seminal work [4], proposed a communication model for DMCs with feedback where the blocklength $\tau \in \mathbb{N}$ is a random variable whose expectation is over bounded by some positive real number $N \in \mathbb{R}_{+}$. He demonstrated that the reliability function or optimal error exponent for the DMC with feedback improves dramatically over the no feedback case and the case where the blocklength is deterministic. This class of codes is known as variable-length codes with feedback. In fact, the reliability function of a DMC with variable-length feedback admits the particularly simple expression

$$
\mathrm{E}(R)=B\left(1-\frac{R}{C}\right), \quad \forall R \in[0, C],
$$

where $C$ is the capacity of the $\mathrm{DMC}$ and $B$ (usually written as $C_{1}$ in the literature) is the relative entropy between conditional output distributions of the two most "most distinguisable" channel input symbols [4]. Yamamoto and Itoh [8] proposed a simple and conceptually important two-phase coding scheme that achieves the reliability function in (1). Burnashev [9] later extended the ideas in [4] to be amenable to the more general problem of sequential hypothesis testing. In particular, he studied the minimum expected number of observations (transmissions) to attain some level of reliability and found the reliability function for large class of single-user channels (beyond DMCs), including the Gaussian channel [9]. Berlin et al. [10] provided a simple converse proof for Burnashev's reliability function [4] that parallels Yamamoto and Itoh's two-phase achievability scheme. Nakiboğlu and Gallager [11] investigated variable-length coding schemes for (not necessarily discrete) memoryless channels with variable-length feedback and with cost constraints and established the reliability function. Mahajan and Tatikonda [12] considered the variable-length case for compound channels [13] and established inner and outer bounds on the so-called error exponent region. Tchamkerten and Telatar, in a series of elegant works [14]-[16], considered conditions in which one can achieve Burnashev's exponent in (1) universally, i.e., without precise knowledge of the DMC.

For the above-mentioned works, we assume that the transmission rate is a fixed value below the channel capacity. It is natural to ask what happens in other regimes. One of the other regimes that has gained interest recently is the second-order regime in which the average error probability of the code $\epsilon>0$ is non-vanishing. Polyanskiy, Poor and Verdú [5] showed that

The authors are with the Department of Electrical and Computer Engineering, National University of Singapore (NUS). V. Y. F. Tan is also with the Department of Mathematics, NUS. Emails: lantruong@u.nus.edu; vtan@nus.edu.sg 
$\epsilon$-capacity is enhanced by a factor of $\frac{1}{1-\epsilon}$ and optimum codes approach the $\epsilon$-capacity rapidly—at a rate of $O\left(\frac{\ln N}{N}\right)$. Kostina, Polyanskiy, and Verdú [17] recently extended this framework to the joint source-channel coding scenario.

We analyze variable-length codes that operate in a regime that is "sandwiched between" the error exponent and secondorder regimes so the codes have vanishing error probabilities and approach capacity as the lengths of the codes grow. This is known as the moderate deviations regime. Here, we mention a few existing works on moderate deviations analysis in information theory. Chen et al. [18] and He et al. [19] studied the moderate deviation asymptotics for fixed-to-variable length source coding with decoder side information and cyclic symmetric channels. Altuğ and Wagner [6] established the moderate deviations constant for DMCs by considering the behavior of the random coding and sphere packing exponents near capacity. Polyanksiy and Verdú [7] provided a different derivation using the information spectrum method and they also considered Gaussian channels. In [6] and [7], it is shown that for a backoff from capacity of $\rho_{n}>0$ (where $\rho_{n}$ decays slower than the reciprocal of square root of the blocklength $n$ ), the error probability decays sub-exponentially with speed $\exp \left(-n \rho_{n}^{2} /(2 V)\right)$ where $V$ is the dispersion of the channel. Tan [20] and Altuğ, Wagner and Kontoyiannis [21] considered moderate deviations for lossy and lossless source coding respectively. Altŭg, Poor and Verdú [22] studied the moderate deviations behavior for fixed-length channel codes with feedback. The authors showed that, under some conditions on DMCs [22, Corollary 1], the moderate deviations constant $1 /(2 \mathrm{~V})$ remains unchanged. In all works on moderate deviation asymptotics for fixed-length block codes, the error probability scales as $\exp \left(-\Theta\left(n \rho_{n}^{2}\right)\right)$.

\section{B. Main Contributions}

We show that for variable-length codes with feedback in the moderate deviations regime, the error probability scales as $\exp \left(-\Theta\left(N \rho_{N}\right)\right)$, where $N$ is the expectation of the (random) blocklength and $\rho_{N}=\omega(1 / \sqrt{N})$. Moreover the implied constant in the $\Theta(\cdot)$ notation, known as the optimal moderate deviations constant, is $B / C$. This is not overly surprising in light of Burnashev's result in (1) because if we take $R$ therein to be $R=C-\rho_{N}$, we obtain

$$
\mathrm{E}\left(C-\rho_{N}\right)=B\left(1-\frac{C-\rho_{N}}{C}\right)=\frac{B}{C} \cdot \rho_{N} .
$$

Hence, we expect the optimum error probability at expected blocklength $N$ to behave as $\exp \left(-(B / C) N \rho_{N}\right)$. Note that the "exponent" here contains $\rho_{N}$ instead of $\rho_{n}^{2}$ (for fixed-length codes as discussed in Section I-A) so this is further evidence that variable-length codes dramatically improve the error probability performance over fixed-length codes. This phenomenon has also been observed in other contexts such as decoding with the erasure option [23, Theorems $1 \& 3$ ] and streaming communications with variable decoding delay [24, Theorem 7]. This derivation in (2) is, of course, heuristic and non-rigorous. This paper aims to make this derivation precise. The contributions are twofold.

1) Our first contribution, the direct part, is to judiciously modify Burnashev's original coding scheme [4] to achieve (1) so that it is amenable to analysis in the moderate deviations regime. In particular, we derive some new results (e.g., Lemmas 2 and 3) for the stopping times of sequences of random variables with properties that resemble both supermartingales and submartingales. These extensions play important roles to bound the expectations of the stopping times of the codes and thus obtaining the exact moderate deviation constant.

2) Our second contribution, the converse part, consists in supplementing some new real analytical arguments to Burnashev's converse proof in [4]. Compared to the original argument [4], we also simplify the proof technique, which involves the construction of an appropriate submartingale (cf. Lemma 7). We do this by leveraging ideas from Burnashev's sequential hypothesis testing paper [9].

\section{Notational Conventions}

We use asymptotic notation such as $O(\cdot)$ in the standard manner, e.g., $f_{n}=O\left(g_{n}\right)$ holds if $\lim _{\sup _{n \rightarrow \infty}}\left|f_{n} / g_{n}\right|<\infty$. We use $\ln x$ to denote the natural logarithm so information units throughout are in nats. We also define the function $(x)_{a}=x \mathbf{1}\{x \geq a\}$ for $x, a \in \mathbb{R}$. The minimum of two real numbers $a$ and $b$ is denoted interchangeably as $\min \{a, b\}$ and $a \wedge b$. As is usual in information theory, $Z_{i}^{j}$ denotes the random vector $\left(Z_{i}, Z_{i+1}, \ldots, Z_{j}\right)$. We usually write $Z_{1}^{j}$ as $Z^{j}$ for brevity.

For any discrete sample space $\mathcal{Z}$, a $\sigma$-algebra $\mathcal{F}$ on $\mathcal{Z}$, a random variable $Z$, and a regular conditional probability measure $\mathbb{P}(\cdot \mid \mathcal{F})$ on $\mathcal{Z}$, define the random and usual conditional entropies as

$$
\begin{aligned}
\mathcal{H}(Z \mid \mathcal{F}) & =-\sum_{z \in \mathcal{Z}} \mathbb{P}(z \mid \mathcal{F}) \ln \mathbb{P}(z \mid \mathcal{F}), \quad \text { and } \\
H(Z) & =\mathcal{H}(Z \mid \sigma(\emptyset, \mathcal{Z})) .
\end{aligned}
$$

If $\mathcal{F}=\sigma\left(Y^{n}\right)$ for some vector $Y^{n}$, we write $\sigma\left(Y^{n}\right)$ as $Y^{n}$ in (3) for simplicity [25].

\section{Organization of the Paper}

The rest of this paper is structured as follows. In Section II, we provide a precise problem statement for DMCs with variablelength codes with feedback and we state the main result. The achievability proof is provided in Section III, and the converse proof is provided in Section IV. Technical derivations are relegated to the appendices. 


\section{Problem Definition And Main Result}

Definition 1. $A(M, N)$-variable-length feedback (VLF) code for a DMC $P_{Y \mid X}$, where $N$ is a positive real and $M$ is a positive integer, is defined by

- A set of equiprobable messages $\mathcal{W}=\{1,2, \ldots, M\}$.

- A sequence of encoders $\left\{f_{n}: \mathcal{W} \times \mathcal{Y}^{n-1} \rightarrow \mathcal{X}\right\}_{n \geq 1}$, defining channel inputs $X_{n}=f_{n}\left(W, Y^{n-1}\right)$.

- A sequence of decoders $\left\{g_{n}: \mathcal{Y}^{n} \rightarrow \mathcal{W}\right\}_{n \geq 1}$, providing estimates of $W$ at various times $n$ at the decoder.

- A random variable $\tau$, which is a stopping time of the filtration $\left\{\sigma\left(Y^{n}\right)\right\}_{n=0}^{\infty}$. Furthermore, $\tau$ satisfies

$$
\mathbb{E}(\tau) \leq N
$$

The final decision at the decoder is computed at time $\tau$ as follows: $\hat{W}=g_{\tau}\left(Y^{\tau}\right)$. The average error probability of a given $\left(M_{N}, N\right)$-VLF code with rate $R_{N}=\frac{1}{N} \ln M_{N}$ is defined as $\mathrm{P}_{\mathrm{e}}\left(R_{N}, N\right)=\mathbb{P}(\hat{W} \neq W)$.

Definition 2. The number $E \geq 0$ is an achievable moderate deviations constant if there exists a family (indexed by $N \in \mathbb{R}_{+}$) of $\left(M_{N}, N\right)-V L F$ codes with $M_{N}=\exp \left(N R_{N}\right)$ and a family of positive real numbers $\left\{\rho_{N}\right\}_{N \in \mathbb{R}_{+}}$satisfying $R_{N} \geq C-\rho_{N}$ and

$$
\begin{aligned}
\lim _{N \rightarrow \infty} \rho_{N} & =0, \\
\lim _{N \rightarrow \infty} \rho_{N} \sqrt{N} & =\infty, \\
\lim _{N \rightarrow \infty} \mathrm{P}_{\mathrm{e}}\left(R_{N}, N\right) & =0, \\
\liminf _{N \rightarrow \infty}-\frac{\ln \mathrm{P}_{\mathrm{e}}\left(R_{N}, N\right)}{N \rho_{N}} & \geq E .
\end{aligned}
$$

We define the (optimal) moderate deviations constant $E^{*}$ (which implicitly depends on $\left\{\rho_{N}\right\}_{N \in \mathbb{R}_{+}}$and $P_{Y \mid X}$ ) as the supremum of all achievable moderate deviations constants.

Definition 3. For a given DMC $P_{Y \mid X}$, we define

$$
\begin{aligned}
B & =\max _{x, x^{\prime} \in \mathcal{X}} D\left(P_{Y \mid X}(\cdot \mid x) \| P_{Y \mid X}\left(\cdot \mid x^{\prime}\right)\right), \\
B^{*} & =\max _{\substack{x, x^{\prime} \in \mathcal{X} \\
D\left(P_{Y \mid X}(\cdot \mid x) \| P_{Y \mid X}\left(\cdot \mid x^{\prime}\right)\right)=B}} D\left(P_{Y \mid X}\left(\cdot \mid x^{\prime}\right) \| P_{Y \mid X}(\cdot \mid x)\right) \\
C & =\max _{P_{X}} I(X ; Y), \\
T & =\max _{x, x^{\prime} \in \mathcal{X}, y \in \mathcal{Y}} \frac{P_{Y \mid X}(y \mid x)}{P_{Y \mid X}\left(y \mid x^{\prime}\right)}, \\
C_{2} & =\max _{x, x^{\prime} \in \mathcal{X}, y \in \mathcal{Y}}\left|\ln \frac{P_{Y \mid X}(y \mid x)}{P_{Y \mid X}\left(y \mid x^{\prime}\right)}\right| .
\end{aligned}
$$

The main result of this paper is stated in the following theorem.

Theorem 1. Assume that there exists a capacity-achieving input distribution $P_{X}^{*}$ for the DMC $P_{Y \mid X}$ satisfying $\min _{x \in \mathcal{X}} P_{X}^{*}(x)>$ 0. Then for $B<\infty$, the following holds

$$
E^{*}=\frac{B}{C} \text {. }
$$

Remark 1. Some remarks are in order.

- For the case $B<\infty$, all quantities defined in Definition 3 are finite.

- For the case $B=\infty$, Burnashev [4, Section 6] proved the existence of an $(M, N)$-VLF code with zero error probability for any $M, N$ by choosing an appropriate pair of input symbols for the hypothesis testing phase of the proposed coding scheme. This fact obviously means that $E^{*}=\infty$ when $B=\infty$.

- The conclusion of Theorem 1 remains the same if the requirement on $\rho_{N}$ in (7) is relaxed to $\rho_{N} N^{t} \rightarrow \infty$ for any $t \in(0,1)$ (instead of restricting to $t=1 / 2$ in (7)). For example, we may take $\rho_{N}=N^{-s}$ for any $s \in(0,1)$. Theorem 1 and (9) then ensure that error probability $\mathrm{P}_{\mathrm{e}}\left(R_{N}, N\right)$ is approximately $\exp \left(-\Theta\left(N^{1-s}\right)\right)$. However, this does not include all subexponential functions such as decaying polynomials in which $\mathrm{P}_{\mathrm{e}}\left(R_{N}, N\right) \approx N^{-\kappa}$ for some $\kappa>0$. We state (7) as it is for notational simplicity in the proof.

The achievability and converse proofs of Theorem 1 are provided in Sections III and IV respectively. 


\section{Achievability Proof}

We start with four preliminary lemmas before providing the achievability proof of Theorem 1 .

Lemma 1. Let $K_{1}, K_{2}, K_{3}$ be three positive numbers and let the sequence $\left\{\xi_{n}\right\}_{n=1}^{\infty}$ be a submartingale adapted to the filtration $\left\{\mathcal{F}_{n}\right\}_{n=1}^{\infty}$, and $\xi_{0} \in \mathbb{R}$ is a constant. In addition, assume that

$$
\begin{aligned}
& \mathbb{E}\left(\xi_{n+1} \mid \mathcal{F}_{n}\right) \geq \xi_{n}+K_{1}, \quad \text { if } \quad \xi_{n}<0, \\
& \mathbb{E}\left(\xi_{n+1} \mid \mathcal{F}_{n}\right) \geq \xi_{n}+K_{2}, \quad \text { if } \quad \xi_{n} \geq 0, \\
&\left|\xi_{n+1}-\xi_{n}\right| \leq K_{3},
\end{aligned}
$$

and the stopping time $\tau$ is given by

$$
\tau=\inf \left\{n: \xi_{n} \geq T\right\}
$$

for some $T \in \mathbb{R}$. Then, we have

$$
\mathbb{E}(\tau) \leq K_{2}^{-1}|T|-K_{1}^{-1} \xi_{0} \mathbf{1}\left\{\xi_{0}<0\right\}-K_{2}^{-1} \xi_{0} \mathbf{1}\left\{\xi_{0} \geq 0\right\}+f\left(K_{1}, K_{2}, K_{3}\right),
$$

where the function $f$ depends only on $K_{1}, K_{2}$, and $K_{3}$.

Proof of Lemma 1: Please see Appendices A and B.

Remark 2. Some remarks concerning Lemma 1 are in order.

- This lemma is an extension of [4, Lemma 6] and [26, Lemma 1] to account for a wider range of parameters. It is proved by Naghshvar, Javidi, and Wigger for the case $T>0$ [27, Lemma 8] and without the constraint (18). However, we provide an alternative proof that holds for all $T \in \mathbb{R}$ which uses a different construction of a submartingale (compared to [26], [27]). We also show rigorously that $\tau$ is almost surely finite in Lemma 13, which is essential for the proof of Lemma 1 and of Lemmas 2 and 3 to follow.

- Moreover, the reason why $\xi_{\tau}$ is a well-defined random variable was not provided in [4], [26], and [27]. We prove this rigorously in Lemma 14 in Appendix B by showing that (i) $\xi_{\tau}$ is a measurable function (due in part to the a.s. finiteness of $\tau$ ) and (ii) $\xi_{\tau} \in L^{1}(\mathbb{R})$ (i.e., $\mathbb{E}\left(\left|\xi_{\tau}\right|\right)$ exists and is finite).

- This lemma, together with Lemmas 2 and 3 to follow, is important in bounding the expected lengths of the constructed VLF codes.

Lemma 2. Let $K_{1}, K_{2}, K_{3}$ be three positive numbers and let the sequence of random variables $\left\{\xi_{n}\right\}_{n=1}^{\infty}$ be adapted to a filtration $\left\{\mathcal{F}_{n}\right\}_{n=1}^{\infty}$. In addition, assume that (18) holds and

$$
\begin{aligned}
& \mathbb{E}\left(\xi_{n+1} \mid \mathcal{F}_{n}\right) \geq \xi_{n}+K_{1}, \quad \text { if } \quad n<\tau_{0}, \\
& \mathbb{E}\left(\xi_{n+1} \mid \mathcal{F}_{n}\right) \leq \xi_{n}-K_{2}, \quad \text { if } n \geq \tau_{0},
\end{aligned}
$$

where

$$
\tau_{0}=\inf \left\{n: \xi_{n} \geq T_{0}\right\}
$$

for some $T_{0} \in \mathbb{R}$. Define

$$
\tau=\inf \left\{n \geq \tau_{0}: \xi_{n} \leq T\right\}
$$

for some $T \leq T_{0} \in \mathbb{R}$. Then, the following bound holds

$$
\mathbb{E}\left(\tau-\tau_{0}\right) \leq \frac{\left|T_{0}-T\right|+3 K_{3}}{K_{2}}
$$

Proof of Lemma 2: Please see Appendix C.

Lemma 3. Assume that all the conditions of Lemma 2 hold, except that (22) is replaced by

$$
\mathbb{E}\left(\xi_{n+1} \mid \mathcal{F}_{n}\right) \geq \xi_{n}+K_{2}, \quad \text { if } n \geq \tau_{0},
$$

and (24) is replaced by (19) for some $T \geq T_{0}$ (where $T_{0} \in \mathbb{R}$ is mentioned in Lemma 2). Then, (25) also holds.

Proof of Lemma 3: This is completely parallel to the proof of Lemma 2 (in Appendix C) and hence omitted.

Lemma 4. Assume that $\left\{\rho_{L}^{\prime}\right\}_{L \in \mathbb{R}_{+}}$is a family of positive numbers satisfying

$$
\begin{aligned}
\lim _{L \rightarrow \infty} \rho_{L}^{\prime} & =0, \\
\lim _{L \rightarrow \infty} \rho_{L}^{\prime} \sqrt{L} & =\infty .
\end{aligned}
$$


Recall the definitions of $B, B^{*}, C_{2}$ from (10), (11), and (14). Let $q_{1}\left(P_{Y \mid X}\right)$ be a function that depends on $P_{Y \mid X}$ and let

$$
\begin{aligned}
p_{0, L} & =1-\frac{1}{L} \\
Z_{0, L} & =\ln \left(\frac{p_{0, L}}{1-p_{0, L}}\right) \\
\varepsilon_{L} & =\exp \left\{\frac{B}{p_{0, L}}\left[-\frac{L \rho_{L}^{\prime}}{C}+\left(\frac{1}{C}-\frac{p_{0, L}}{B}+\frac{3\left(1-p_{0, L}\right)}{2 B^{*}}\right) Z_{0, L}+q_{1}\left(P_{Y \mid X}\right)\right]\right\} \\
A_{L} & =\frac{Z_{0, L}}{2} \\
p_{1, L} & \in\left[0, \frac{\exp \left(-Z_{0, L}\right)-\varepsilon_{L} \exp \left(-C_{2}\right)}{\exp \left(-A_{L}\right)-\varepsilon_{L} \exp \left(-C_{2}\right)}\right]
\end{aligned}
$$

In addition, assume that $\left\{W_{L}\right\}_{L \in \mathbb{R}_{+}}$is a family of random variables whose expected values satisfy

$$
\begin{aligned}
p_{0, L}\left(1-p_{1, L}\right) \mathbb{E}\left(W_{L}\right)= & -\frac{p_{0, L} \ln \varepsilon_{L}}{B}+\frac{\ln \left(\exp \left(L\left(C-\rho_{L}^{\prime}\right)\right)-1\right)}{C} \\
& +\left[\frac{1}{C}-\frac{p_{0, L}}{B}+\frac{\left(1-p_{0, L}\right)}{B^{*}}\right] Z_{0, L}+\frac{\left(1-p_{0, L}\right)\left|A_{L}\right|}{B^{*}}+q_{1}\left(P_{Y \mid X}\right) .
\end{aligned}
$$

Then, we have

$$
\begin{aligned}
\liminf _{L \rightarrow \infty}-\frac{\ln \varepsilon_{L}}{L \rho_{L}^{\prime}} & \geq \frac{B}{C} \quad \text { and } \\
\mathbb{E}\left(W_{L}\right) & \leq L+3 \sqrt{L}
\end{aligned}
$$

for L sufficiently large.

Proof of Lemma 4: Please see Appendix D.

Proposition 1 (Achievability of Theorem 1). Under the conditions of Theorem 1,

$$
E^{*} \geq \frac{B}{C}
$$

Remark 3. Some remarks are in order.

- In this achievability proof, our main contribution is to provide a proof for some auxiliary results in [4] to ensure the arguments carry through for the moderate deviations regime. More specifically, in the case $B^{*} \leq C$, Burnashev [4, pp. 260] chose the analogue of $A_{L}$ in (32) to be negative in his coding scheme to achieve the optimal error exponent (1). With this choice, the author stated, without proof, a crucial result [4, Eqn. (5.19)] which is then applied to derive the optimal error exponent. In the moderate deviations regime, $A_{L}=(1 / 2) \ln \left(p_{0, L} /\left(1-p_{0, L}\right)\right)>0$ (cf. (32)) is chosen to achieve the optimal moderate deviations constant. Hence, one of the main contributions here is to provide a state and prove Lemmas 2 and 3 which are essential for the proof of the optimal moderate deviations constant. The different choices of other parameters in (29)-(33) vis-à-vis the error exponent regime [4, Eqn. (5.22)] also affect our analyses.

- It appears (at least to the authors) to be more challenging to adapt Yamamoto-Itoh's coding scheme [8] compared to Burnashev's coding scheme [4] since the retransmission probability (or expected length of variable-length code) is a fixed function of the error probability in the communication phase. However, in Burnashev's coding scheme, we can easily control the tradeoff between the error and retransmission probabilities by tuning the parameter $A_{L}$ [4, pp. 20].

Proof of Proposition 1: We use the same coding scheme as Burnashev [4] but our definitions of stopping times are different. Burnashev's coding scheme consists of two variable-length coding phases. For the sake of completeness, we provide a sketch of his proof and emphasize what we change to make the proof work for the moderate deviations regime.

First we define

$$
Z_{j}(n)=\ln \frac{\mathbb{P}\left(W=j \mid Y^{n}\right)}{1-\mathbb{P}\left(W=j \mid Y^{n}\right)}, \quad j=1,2, \ldots, M,
$$

and $Z(n)=Z_{\mathrm{m}}(n)$, where $\mathrm{m} \in\{1,2, \ldots, M\}$ is the transmitted message. In addition, fix a pair $\left(x_{0}, x_{0}^{\prime}\right) \in \mathcal{X}^{2}$ such that

$$
\begin{aligned}
& D\left(P_{Y \mid X}\left(\cdot \mid x_{0}\right) \| P_{Y \mid X}\left(\cdot \mid x_{0}^{\prime}\right)\right)=B \\
& D\left(P_{Y \mid X}\left(\cdot \mid x_{0}^{\prime}\right) \| P_{Y \mid X}\left(\cdot \mid x_{0}\right)\right)=B^{*} .
\end{aligned}
$$


Case 1: $B^{*}>C$ : For this case, without loss of generality we can assume that there exists a capacity-achieving input distribution $\left\{P_{X}^{*}(x)\right\}_{x \in \mathcal{X}}$ such that $P_{X}^{*}(x)>0$ for all $x \in \mathcal{X} .{ }^{1}$ Then, we have [28, Theorem 4.5.1] the equality $C=$ $D\left(P_{Y \mid X}(\cdot \mid x) \| \sum_{x^{\prime}} P_{X}^{*}\left(x^{\prime}\right) P_{Y \mid X}\left(\cdot \mid x^{\prime}\right)\right)$ for all $x \in \mathcal{X}$. Define the function, the set, and the constant

$$
\begin{aligned}
\psi(u, v) & :=\sum_{y \in \mathcal{Y}} P_{Y \mid X}\left(y \mid x_{0}^{\prime}\right) \ln \frac{P_{Y \mid X}\left(y \mid x_{0}^{\prime}\right)(1-v)}{P_{Y \mid X}\left(y \mid x_{0}\right)(1-v)+u\left(P_{Y \mid X}\left(y \mid x_{0}\right)-P_{Y \mid X}\left(y \mid x_{0}^{\prime}\right)\right)}, \\
\mathcal{S} & :=\left\{(u, v): \frac{1}{2} \leq u \leq 1,0 \leq v \leq 1-u, \psi(u, v)=C\right\}, \quad \text { and } \\
p_{0} & :=\min \{u:(u, v) \in \mathcal{S} \text { for some } v \in[0,1 / 2]\} .
\end{aligned}
$$

1) Encoding:

- Phase 1: Before transmission at the $(n+1)$-instant, all messages $W \in\{1,2, \ldots, M\}$ are randomly partitioned into $|\mathcal{X}|$ groups $\mathcal{M}_{1}, \mathcal{M}_{2}, \ldots, \mathcal{M}_{|\mathcal{X}|}$. Here, the probability that message $j \in\{1,2, \ldots, M\}$ will be assigned to the group $\mathcal{M}_{x}$ is $\alpha_{j, n}^{x} / \mathbb{P}\left(W=j \mid Y^{n}\right)$, where $\alpha_{j, n}^{x}$ (the fraction of $\mathbb{P}\left(W=j \mid Y^{n}\right)$ corresponding to $\left.P_{X}^{*}(x)\right)$ is defined as

$$
\alpha_{j, n}^{x}:=P_{X}^{*}(x) \mathbb{P}\left(W=j \mid Y^{n}\right),
$$

for each $x \in \mathcal{X}$ and $j \in \mathcal{M}$. It follows from (44) that $\sum_{x \in \mathcal{X}} \alpha_{j, n}^{x}=\mathbb{P}\left(W=j \mid Y^{n}\right)$ and $\sum_{j=1}^{M} \alpha_{j, n}^{x}=P_{X}^{*}(x)$. The stopping time for phase 1 is defined as

$$
\tau_{0}^{*}=\inf \left\{n: \max _{j \in \mathcal{W}} \mathbb{P}\left(W=j \mid Y^{n}\right) \geq p_{0}\right\} .
$$

- Phase 2: At the stopping time $\tau_{0}^{*}$, the posterior probability of one of the messages first exceeds $p_{0}$, i.e. $\mathbb{P}\left(W=j_{0} \mid Y^{n}\right) \geq p_{0}$ for some $j_{0} \in \mathcal{W}$. Then we subsequently solve a problem of discriminating between two hypotheses: $\mathrm{H}_{0}=\left\{j_{0}\right.$ is the true message $\}$ and $\mathrm{H}_{1}=\left\{j_{0}\right.$ is a false message $\}$. Here, $\mathrm{H}_{0}$ is placed in correspondence with input symbol $x_{0}$, while $\mathrm{H}_{1}$ is placed in correspondence with another symbol $x_{0}^{\prime}$, where the pair $\left(x_{0}, x_{0}^{\prime}\right)$ is chosen in (39) and (40).

The stopping time $\tau^{*}$ of the phase 2 (or overall coding scheme) is defined as following:

$$
\tau^{*}=\inf \left\{n \geq 1: \max _{j \in \mathcal{W}} Z_{j}(n) \geq-\ln \varepsilon_{N}\right\}
$$

for a family of real numbers $\left\{\varepsilon_{N}\right\}_{N \in \mathbb{R}_{+}}$to be determined later; see (58) to follow.

2) Decoding: The decoding is performed at the stopping time $\tau^{*}$, and the estimated message is

$$
\hat{W}=\underset{k \in \mathcal{W}}{\arg \max } Z_{k}\left(\tau_{0}^{*}\right)
$$

where $\tau_{0}^{*}$ is defined in (45).

3) Moderate Deviations Analysis: The error probability $\mathrm{P}_{\mathrm{e}}\left(R_{N}, N\right)$ of this coding scheme can be shown to be bounded above by $\varepsilon_{N}$ [4]. Moreover, for $B^{*}>C$, Burnashev [4] proved that

$$
\begin{aligned}
\mathbb{E}\left[Z(n+1)-Z(n) \mid Y^{n}\right] & \geq C, \quad \text { if } \quad Z(n)<\ln \frac{p_{0}}{1-p_{0}}, \\
\mathbb{E}\left[Z(n+1)-Z(n) \mid Y^{n}\right] & =B, \quad \text { if } \quad Z(n) \geq \ln \frac{p_{0}}{1-p_{0}}, \\
|Z(n+1)-Z(n)| & \leq C_{2} .
\end{aligned}
$$

Now, define

$$
\tau_{\mathrm{m}}=\inf \left\{n: Z(n) \geq-\ln \varepsilon_{N}\right\} .
$$

Then, applying Lemma 1 with the identifications $K_{1}=C<K_{2}=B$, and $K_{3}=C_{2}$ for the submartingale $\left\{\xi_{n}\right\}_{n=0}^{\infty}=$ $\left\{Z(n)-\ln \frac{p_{0}}{1-p_{0}}\right\}_{n=0}^{\infty}$ adapted to the filtration $\left\{\mathcal{F}_{n}\right\}_{n=0}^{\infty}=\left\{\sigma\left(Y^{n}\right)\right\}_{n=0}^{\infty}$ with stopping time $\tau_{\mathrm{m}}$ and $T=-\ln \varepsilon_{N}-\ln \frac{p_{0}}{1-p_{0}}$, we obtain

$$
\mathbb{E}\left(\tau_{\mathrm{m}}\right) \leq B^{-1}\left|-\ln \varepsilon_{N}-\ln \frac{p_{0}}{1-p_{0}}\right|+C^{-1}\left|Z(0)-\ln \frac{p_{0}}{1-p_{0}}\right|+h\left(C, B, C_{2}\right),
$$

\footnotetext{
${ }^{1}$ For the first coding phase, Burnashev [4] showed that there exists a coding scheme such that $\mathbb{E}\left[Z(n+1)-Z(n) \mid Y^{n}\right] \geq C, n \in \mathbb{N}$ for any capacity-input distribution. This constitutes one of two key results for the purpose of analyzing the average error probability. The other key result states that $\mathbb{E}[Z(n+$ 1) $\left.-Z(n) \mid Y^{n}\right] \geq B, n \in \mathbb{N}$ holds for the hypothesis testing phase. The assumption that such a capacity-achieving input distribution (with full support) exists makes our notation simpler, i.e, we don't need to change $\mathcal{X}$ to $\mathcal{X}^{\prime}$ where $\mathcal{X}^{\prime}$ is the set of all $x \in \mathcal{X}$ such that $P_{X}^{*}(x)>0$. Note that $C=$ $D\left(P_{Y \mid X}(\cdot \mid x) \| \sum_{x^{\prime}} P_{X}^{*}\left(x^{\prime}\right) P_{Y \mid X}\left(\cdot \mid x^{\prime}\right)\right)$ holds for all $x \in \mathcal{X}^{\prime}$.
} 
where $h\left(C, B, C_{2}\right)$ is a function of $P_{Y \mid X}$. Note that the bound in (52) holds because

$$
Z(0)=\ln \frac{1 / M}{1-1 / M}=-\ln (M-1) \leq 0 \leq \ln \frac{p_{0}}{1-p_{0}},
$$

and $p_{0} \geq 1 / 2$ from (43) (so $\ln \frac{p_{0}}{1-p_{0}} \geq 0$ ).

Now, define new stopping times

$$
t_{j}=\inf \left\{n: Z_{j}(n) \geq-\ln \varepsilon_{N}\right\}, \quad \forall j \in \mathcal{W} .
$$

Then, clearly we have

$$
\tau^{*}=\min _{j \in \mathcal{W}} t_{j}
$$

Now, choose the family of positive numbers $\left\{\varepsilon_{N}\right\}_{N \in \mathbb{R}_{+}}$such that

$$
-C^{-1} N \rho_{N}-B^{-1} \ln \varepsilon_{N}+q_{2}\left(P_{Y \mid X}\right)=0,
$$

where $q_{2}\left(P_{Y \mid X}\right)$ is defined as

$$
q_{2}\left(P_{Y \mid X}\right):=C^{-1} \ln \frac{p_{0}}{1-p_{0}}-B^{-1} \ln \frac{p_{0}}{1-p_{0}}+h\left(C, B, C_{2}\right),
$$

which is a function of the DMC $P_{Y \mid X}$. We note that since $\varepsilon_{N} \rightarrow 0$, the absolute value in the first term on the right-hand-side of (52) can be removed and that $\ln \frac{p_{0}}{1-p_{0}}$ is a constant. Note that (56) is equivalent to choosing

$$
\varepsilon_{N}=\exp \left[-\frac{B}{C} N \rho_{N}+B q_{2}\left(P_{Y \mid X}\right)\right], \quad \forall N \in \mathbb{R}_{+} .
$$

With these choices, we have

$$
\lim _{N \rightarrow \infty} \mathrm{P}_{\mathrm{e}}\left(R_{N}, N\right) \leq \lim _{N \rightarrow \infty} \varepsilon_{N}=0
$$

Therefore, we conclude that

$$
\liminf _{N \rightarrow \infty} \frac{-\ln \mathrm{P}_{\mathrm{e}}\left(R_{N}, N\right)}{N \rho_{N}} \geq \liminf _{N \rightarrow \infty} \frac{-\ln \varepsilon_{N}}{N \rho_{N}}=\frac{B}{C} .
$$

We obtain for $N$ sufficiently large that stopping time $\tau^{*}$ satisfies

$$
\begin{aligned}
\mathbb{E}\left(\tau^{*}\right) & =\mathbb{E}\left(\min _{j \in \mathcal{W}} t_{j}\right) \\
& \leq \mathbb{E}\left(\tau_{\mathrm{m}}\right) \\
& \leq C^{-1} \ln M-B^{-1} \ln \varepsilon_{N}+q_{2}\left(P_{Y \mid X}\right) \\
& =N-C^{-1} N \rho_{N}-B^{-1} \ln \varepsilon_{N}+q_{2}\left(P_{Y \mid X}\right) \\
& =N
\end{aligned}
$$

where (61) follows from (55), (63) follows from (52), (53) and (59) with $q_{2}\left(P_{Y \mid X}\right)$ to be defined in (57), (64) follows from $\log M=N R_{N}=N\left(C-\rho_{N}\right)$, and (65) follows from (56). Thus, the constraint on the expectation of the stopping time is satisfied. This and (60) complete the proof for the case $B^{*}>C$.

Case 2: $B^{*} \leq C$ : For this case, we first show that there exists an $\left(\exp \left(L\left(C-\rho_{L}^{\prime}\right)\right), L+3 \sqrt{L}\right)$-VLF code with the average error probability $\mathrm{P}_{\mathrm{e}}^{\prime}\left(R_{L}, L\right)$ satisfying

$$
\liminf _{L \rightarrow \infty} \frac{-\ln \mathrm{P}_{\mathrm{e}}^{\prime}\left(R_{L}, L\right)}{L \rho_{L}^{\prime}} \geq \frac{B}{C}
$$

for any family of positive numbers $\left\{\rho_{L}^{\prime}\right\}_{L \in \mathbb{R}_{+}}$satisfying (27) and (28). Then we augment the proof to show that $E^{*} \geq B / C$.

To show (66), we modify some choices of important parameters in Burnashev's coding scheme [4]. These modifications require us to extend some key mathematical results in Burnashev's paper to more general settings (cf. Lemmas 1, 2, and 3) so they can be applied to the moderate deviations regime.

As in Burnashev [4], for the case $B^{*} \leq C$, we define a two-phase encoding scheme is as follows.

1) Encoding:

- Phase 1: If prior to instant $n$, the posterior probabilities of all messages $W \in\left\{1,2, \ldots, \exp \left(L\left(C-\rho_{L}^{\prime}\right)\right)\right\}$ are less than $p_{0, L} \in[1 / 2,1)$ defined in (29), the transmission method at the time $n+1$ is the same as the case $B^{*}>C$ in Case 1 above. The stopping time for phase 1 is defined as

$$
\tau_{0}^{*}=\inf \left\{n: \max _{j \in \mathcal{W}} \mathbb{P}\left(W=j \mid Y^{n}\right) \geq p_{0, L}\right\}
$$


where $p_{0, L} \geq 1 / 2$ is defined in (29).

- Phase 2: At the stopping time $\tau_{0}^{*}$, the posterior probability of one of the messages, indexed by $j_{0} \in \mathcal{W}$, first exceeds $p_{0, L}$, i.e. $\mathbb{P}\left(W=j_{0} \mid Y^{n}\right) \geq p_{0, L}$ where $p_{0, L} \geq 1 / 2$ is defined in (29). Then we subsequently solve a problem of discriminating between two hypotheses: $\mathrm{H}_{0}=\left\{j_{0}\right.$ is the true message $\}$ and $\mathrm{H}_{1}=\left\{j_{0}\right.$ is a false message $\}$. Here, $\mathrm{H}_{0}$ is placed in correspondence with input symbol $x_{0}$, while $\mathrm{H}_{1}$ is placed in correspondence with another symbol $x_{0}^{\prime}$, where the pair $\left(x_{0}, x_{0}^{\prime}\right)$ is chosen in (39) and (40).

The stopping time of the phase 2 (or the overall coding scheme) is defined as follows:

$$
\tau^{*}=\tau_{1}^{*} \wedge \tau_{2}^{*}
$$

where

$$
\begin{aligned}
& \tau_{1}^{*}=\inf \left\{n \geq \tau_{0}^{*}: \max _{j \in \mathcal{W}} Z_{j} \geq-\ln \varepsilon_{L}\right\}, \\
& \tau_{2}^{*}=\inf \left\{n \geq \tau_{0}^{*}: \min _{j \in \mathcal{W}} Z_{j} \leq A_{L}\right\}
\end{aligned}
$$

where $Z_{0, L}$ and $A_{L}$ are defined in (30) and (32) respectively. It is easy to see from the definition of $\tau_{0}^{*}$ in (67) and the definition of $Z_{0, L}$ in (30) that

$$
\tau_{0}^{*}=\inf \left\{n: \max _{j \in \mathcal{W}} Z_{j}(n) \geq Z_{0, L}\right\}
$$

If $\tau_{1}^{*}>\tau_{2}^{*}$, we retransmit the message.

2) Decoding: If $\tau_{1}^{*} \leq \tau_{2}^{*}$, so $\tau^{*}=\tau_{1}^{*}$, the message is decoded at time $\tau^{*}$ and the decoding is performed as follows

$$
\hat{W}=\underset{k \in \mathcal{W}}{\arg \max } Z_{k}\left(\tau_{0}^{*}\right)
$$

where $\tau_{0}^{*}$ is defined in (71).

3) Moderate Deviations Analysis: The error probability of this coding scheme $\mathrm{P}_{\mathrm{e}}^{\prime}\left(R_{L}, L\right)$ can be shown to be bounded above by $\varepsilon_{L}$ [4]. Assume that $\mathrm{m}$ is the transmitted message. Using this coding scheme, Burnashev [4] also showed that

$$
\begin{aligned}
\mathbb{E}\left[Z(n+1)-Z(n) \mid Y^{n}\right] & \geq C, \\
\left|Z_{j}(n+1)-Z_{j}(n)\right| & \leq C_{2}, \quad, \quad \forall j \in \mathcal{W}, \\
\mathbb{E}\left[Z(n+1)-Z(n) \mid Y^{n}\right] & =B, \quad \text { if } n \geq \tau_{0}^{*}, \quad \text { under } \quad \mathrm{H}_{0}, \\
\mathbb{E}\left[Z_{j_{0}}(n+1)-Z_{j_{0}}(n) \mid Y^{n}\right] & =-B^{*}, \quad \text { if } n \geq \tau_{0}^{*}, \quad \text { under } \quad \mathrm{H}_{1} .
\end{aligned}
$$

Let $\left\{Z_{0, L}\right\}_{L \in \mathbb{R}^{+}}$and $\left\{A_{L}\right\}_{L \in \mathbb{R}^{+}}$be the families of real numbers defined as in (30) and (32) respectively. Define

$$
\begin{aligned}
& \tau_{0 \mathrm{~m}}=\inf \left\{n \geq 0: Z(n) \geq Z_{0, L}\right\}, \\
& \tau_{1 \mathrm{~m}}=\inf \left\{n \geq \tau_{0 \mathrm{~m}}: Z(n) \geq-\ln \varepsilon_{L}\right\}, \\
& \tau_{2 \mathrm{~m}}=\inf \left\{n \geq \tau_{0 \mathrm{~m}}: Z(n) \leq A_{L}\right\}, \\
& \tau_{0 \mathrm{~m}}^{-}=\inf \left\{n \geq 0: \max _{j \in \mathcal{W} \backslash\{\mathrm{m}\}} Z_{j}(n) \geq Z_{0, L}\right\}, \\
& \tau_{1 \mathrm{~m}}^{-}=\inf \left\{n \geq \tau_{0 \mathrm{~m}}^{-}: \max _{j \in \mathcal{W} \backslash\{\mathrm{m}\}} Z_{j}(n) \geq-\ln \varepsilon_{L}\right\}, \\
& \tau_{2 \mathrm{~m}}^{-}=\inf \left\{n \geq \tau_{0 \mathrm{~m}}^{-}: \min _{j \in \mathcal{W} \backslash\{\mathrm{m}\}} Z_{j}(n) \leq A_{L}\right\}, \\
& \tau_{0 j_{0}}=\inf \left\{n \geq 0: Z_{j 0}(n) \geq Z_{0, L}\right\}, \\
& \tau_{2 j_{0}}=\inf \left\{n \geq \tau_{0 j_{0}}: Z_{j_{0}}(n) \leq A_{L}\right\} .
\end{aligned}
$$

Let $p_{1, L}=\mathbb{P}\left(\tau_{2 \mathrm{~m}}<\tau_{1 \mathrm{~m}}\right)$. Using this coding scheme, the retransmission probability ${ }^{2}$ can be easily obtained as

$$
\mathrm{P}_{\mathrm{x}}=1-p_{0, L}\left(1-p_{1, L}\right)
$$

\footnotetext{
${ }^{2}$ In the coding scheme, the message will be retransmitted under the condition $\tau_{1}^{*}>\tau_{2}^{*}$ as stated after (71).
} 
It follows that the expected value of the stopping time of the overall coding scheme $\mathbb{E}\left(\tau^{*}\right)$ satisfies

$$
\begin{aligned}
\left(1-\mathrm{P}_{\mathrm{x}}\right) \mathbb{E}\left(\tau^{*}\right) & =p_{0, L} \mathbb{E}\left(\tau^{*} \mid \mathrm{H}_{0}\right)+\left(1-p_{0, L}\right) \mathbb{E}\left(\tau^{*} \mid \mathrm{H}_{1}\right) \\
& \leq p_{0, L} \mathbb{E}\left(\tau_{1}^{*} \mid \mathrm{H}_{0}\right)+\left(1-p_{0, L}\right) \mathbb{E}\left(\tau_{2}^{*} \mid \mathrm{H}_{1}\right) \\
& =p_{0, L} \mathbb{E}\left(\tau_{0}^{*} \mid \mathrm{H}_{0}\right)+\left(1-p_{0, L}\right) \mathbb{E}\left(\tau_{0}^{*} \mid \mathrm{H}_{1}\right)+p_{0, L} \mathbb{E}\left(\tau_{1}^{*}-\tau_{0}^{*} \mid \mathrm{H}_{0}\right)+\left(1-p_{0, L}\right) \mathbb{E}\left(\tau_{2}^{*}-\tau_{0}^{*} \mid \mathrm{H}_{1}\right) \\
& =p_{0, L} \mathbb{E}\left(\tau_{0 \mathrm{~m}} \mid \mathrm{H}_{0}\right)+\left(1-p_{0, L}\right) \mathbb{E}\left(\tau_{0 \mathrm{~m}}^{-} \mid \mathrm{H}_{1}\right)+p_{0, L} \mathbb{E}\left(\tau_{1}^{*}-\tau_{0 \mathrm{~m}} \mid \mathrm{H}_{0}\right)+\left(1-p_{0, L}\right) \mathbb{E}\left(\tau_{2}^{*}-\tau_{0 \mathrm{~m}}^{-} \mid \mathrm{H}_{1}\right) \\
& \leq p_{0, L} \mathbb{E}\left(\tau_{0 \mathrm{~m}} \mid \mathrm{H}_{0}\right)+\left(1-p_{0, L}\right) \mathbb{E}\left(\tau_{0 \mathrm{~m}}^{-} \mid \mathrm{H}_{1}\right)+p_{0, L} \mathbb{E}\left(\tau_{1 \mathrm{~m}}-\tau_{0 \mathrm{~m}} \mid \mathrm{H}_{0}\right)+\left(1-p_{0, L}\right) \mathbb{E}\left(\tau_{2 \mathrm{~m}}^{-}-\tau_{0 \mathrm{~m}}^{-} \mid \mathrm{H}_{1}\right) \\
& \leq p_{0, L} \mathbb{E}\left(\tau_{0 \mathrm{~m}} \mid \mathrm{H}_{0}\right)+\left(1-p_{0, L}\right) \mathbb{E}\left(\tau_{0 \mathrm{~m}} \mid \mathrm{H}_{1}\right)+p_{0, L} \mathbb{E}\left(\tau_{1 \mathrm{~m}}-\tau_{0 \mathrm{~m}} \mid \mathrm{H}_{0}\right)+\left(1-p_{0, L}\right) \mathbb{E}\left(\tau_{2 \mathrm{~m}}^{-}-\tau_{0 \mathrm{~m}}^{-} \mid \mathrm{H}_{1}\right) \\
& =\mathbb{E}\left(\tau_{0 \mathrm{~m}}\right)+p_{0, L} \mathbb{E}\left(\tau_{1 \mathrm{~m}}-\tau_{0 \mathrm{~m}} \mid \mathrm{H}_{0}\right)+\left(1-p_{0, L}\right) \mathbb{E}\left(\tau_{2 \mathrm{~m}}^{-}-\tau_{0 \mathrm{~m}}^{-} \mid \mathrm{H}_{1}\right) \\
& =\mathbb{E}\left(\tau_{0 \mathrm{~m}}\right)+p_{0, L} \mathbb{E}\left(\tau_{1 \mathrm{~m}}-\tau_{0 \mathrm{~m}} \mid \mathrm{H}_{0}\right)+\left(1-p_{0, L}\right) \mathbb{E}\left(\tau_{2 \mathrm{~m}}^{-}-\tau_{0 j_{0}} \mid \mathrm{H}_{1}\right) \\
& \leq \mathbb{E}\left(\tau_{0 \mathrm{~m}}\right)+p_{0, L} \mathbb{E}\left(\tau_{1 \mathrm{~m}}-\tau_{0 \mathrm{~m}} \mid \mathrm{H}_{0}\right)+\left(1-p_{0, L}\right) \mathbb{E}\left(\tau_{2 j_{0}}-\tau_{0 j_{0}} \mid \mathrm{H}_{1}\right) .
\end{aligned}
$$

Here, (87) follows from (68), (89) follows from the fact that under $\mathrm{H}_{0}$ we have $\tau_{0}^{*}=\tau_{0 \mathrm{~m}}$ and that under $\mathrm{H}_{1}$ we have $\tau_{0}^{*}=\tau_{0 \mathrm{~m}}^{-}$, (90) is obtained from the same arguments as in (62), (91) follows from the fact that under $\mathrm{H}_{1}$ we have $\tau_{0 \mathrm{~m}} \geq \tau_{0 \mathrm{~m}}^{-}$, (93) follows from the encoding assumption for Phase 2 that $\tau_{0 j_{0}}=\tau_{0 \mathrm{~m}}^{-}$under $\mathrm{H}_{1}$, and finally (94) follows from the fact that $\tau_{2 j_{0}} \geq \tau_{2 \mathrm{~m}}^{-}$by using the same arguments as in (62).

Therefore, we obtain

$$
p_{0, L}\left(1-p_{1, L}\right) \mathbb{E}\left(\tau^{*}\right) \leq \mathbb{E}\left(\tau_{0 \mathrm{~m}}\right)+p_{0, L} \mathbb{E}\left(\tau_{1 \mathrm{~m}}-\tau_{0 \mathrm{~m}}\right)+\left(1-p_{0, L}\right) \mathbb{E}\left(\tau_{2 j_{0}}-\tau_{0 j_{0}}\right) .
$$

From (73) and (77) we see that in Phase 1, $\{Z(n)\}_{n=0}^{\infty}$ forms a submartingale adapted to the filtration $\left\{\sigma\left(Y^{n}\right)\right\}_{n=0}^{\infty}$ with initial value $Z(0)$ and with stopping time $\tau_{0 \mathrm{~m}}=\inf \left\{n: Z(n) \geq Z_{0, L}\right\}$. Applying Lemma 1 with the identifications $K_{1}=K_{2}=C$ and $K_{3}=C_{2}$ we obtain

$$
\mathbb{E}\left(\tau_{0 \mathrm{~m}}\right) \leq \frac{Z_{0, L}-Z(0)}{C}+\tilde{f}\left(C_{2}, C\right) .
$$

for a function $\tilde{f}$ that depends only on $C_{2}$ and $C$.

In Phase 2, there are two hypotheses.

- Under $\mathrm{H}_{1}$, the sequence

$$
\tilde{Z}_{j_{0}}(n)=\left\{\begin{array}{ll}
Z(n), & \tau<\tau_{0 \mathrm{~m}} \\
Z_{j_{0}}(n), & \tau \geq \tau_{0 \mathrm{~m}}
\end{array},\right.
$$

which is adapted to the filtration $\left\{\sigma\left(Y^{n}\right)\right\}_{n=0}^{\infty}$, satisfies all requirements of Lemma 2 with $\tau_{0}=\tau_{0 j_{0}}, \tau=\tau_{2 j_{0}}, K_{1}=$ $C, K_{2}=B^{*}, K_{3}=C_{2}, T=A_{L}$, and $T_{0}=Z_{0, L}$. Therefore we obtain

$$
\mathbb{E}\left(\tau_{2 j_{0}}-\tau_{0 j_{0}} \mid \mathrm{H}_{1}\right) \leq \frac{Z_{0, L}+\left|A_{L}\right|+3 C_{2}}{B^{*}} .
$$

Note that for $A_{L}>T$, (98) still holds since $\tau_{2 j_{0}}=\tau_{0 j_{0}}$.

- Under $\mathrm{H}_{0}$, the sequence $\left\{\tilde{Z}_{j_{0}}(n)\right\}_{n=0}^{\infty}$, which is adapted to the filtration $\left\{\sigma\left(Y^{n}\right)\right\}_{n=0}^{\infty}$, satisfies all requirements of Lemma 3 with $\tau_{0}=\tau_{0 \mathrm{~m}}, \tau=\tau_{1 \mathrm{~m}}, K_{1}=C, K_{2}=B, K_{3}=C_{2}, T=-\ln \varepsilon_{N}$, and $T_{0}=Z_{0, L}$. Therefore we obtain

$$
\mathbb{E}\left(\tau_{1 \mathrm{~m}}-\tau_{0 \mathrm{~m}} \mid \mathrm{H}_{0}\right) \leq \frac{-\ln \varepsilon_{N}-Z_{0, L}+3 C_{2}}{B} .
$$

Note that

$$
Z(0)=\ln \left(\frac{1 / \exp \left(L\left(C-\rho_{L}^{\prime}\right)\right)}{1-1 / \exp \left(L\left(C-\rho_{L}^{\prime}\right)\right.}\right)=-\ln \left(\exp \left(L\left(C-\rho_{L}^{\prime}\right)\right)-1\right)
$$

We choose

$$
\rho_{L}^{\prime}=\rho_{L+3 \sqrt{L}}-\frac{3}{\sqrt{L}}\left(C-\rho_{L+3 \sqrt{L}}\right) .
$$

It is easy to see that (27) and (28) hold for $\rho_{L}^{\prime}$. In addition, from (95)-(100), the expectation of stopping time $\tau^{*}$ satisfies

$$
\begin{aligned}
p_{0, L}\left(1-p_{1, L}\right) \mathbb{E}\left(\tau^{*}\right)= & -p_{0, L} \frac{\ln \varepsilon_{L}}{B}-\frac{Z(0)}{C}+\left[\frac{1}{C}-\frac{p_{0, L}}{B}+\frac{\left(1-p_{0, L}\right)}{B^{*}}\right] Z_{0, L}+\frac{\left(1-p_{0, L}\right)\left|A_{L}\right|}{B^{*}}+q_{3}\left(P_{Y \mid X}\right) \\
= & -p_{0, L} \frac{\ln \varepsilon_{L}}{B}+\frac{\ln \left(\exp \left(L\left(C-\rho_{L}^{\prime}\right)\right)-1\right)}{C} \\
& +\left[\frac{1}{C}-\frac{p_{0, L}}{B}+\frac{\left(1-p_{0, L}\right)}{B^{*}}\right] Z_{0, L}+\frac{\left(1-p_{0, L}\right)\left|A_{L}\right|}{B^{*}}+q_{3}\left(P_{Y \mid X}\right),
\end{aligned}
$$


where $q_{3}\left(P_{Y \mid X}\right)$ is depends on $P_{Y \mid X}$ and $p_{1, L}=\mathbb{P}\left(\tau_{2 \mathrm{~m}}<\tau_{1 \mathrm{~m}}\right)$ satisfies (33). A special case of (103) was stated without proof in [4, Eqn. (5.19)], where the author assumed that $A_{L}<0$ [4, pp. 260]. The equality (103) is more general as it holds for all $A_{L}$; hence we can set $A_{L}=Z_{0, L} / 2>0$ (cf. (32)). Note that we can easily handle the case $A_{L}>0$ because of our newly-developed Lemmas 2 and 3 leading to (98) and (99) respectively.

By setting $p_{0, L}$ and $\varepsilon_{L}$ as (29) and (31) and applying Lemma 4, we can find an $\left(\exp \left(L\left(C-\rho_{L}^{\prime}\right)\right), L+3 \sqrt{L}\right)$-VLF code such that the average error probability $\mathrm{P}_{\mathrm{e}}^{\prime}\left(R_{L}, L\right)$ satisfies

$$
\liminf _{L \rightarrow \infty} \frac{-\ln \mathrm{P}_{\mathrm{e}}^{\prime}\left(R_{L}, L\right)}{L \rho_{L}^{\prime}} \geq \liminf _{L \rightarrow \infty} \frac{-\ln \varepsilon_{L}}{L \rho_{L}^{\prime}} \geq \frac{B}{C} .
$$

Thus, (66) is shown. Observe from (101) that $\exp \left(L\left(C-\rho_{L}^{\prime}\right)\right)=\exp \left((L+3 \sqrt{L})\left(C-\rho_{L+3 \sqrt{L}}\right)\right)$. Therefore, there exists an $\left(\exp \left((L+3 \sqrt{L})\left(C-\rho_{L+3 \sqrt{L}}\right)\right), L+3 \sqrt{L}\right)-\mathrm{VLF} \operatorname{code}^{3}$ such that

$$
\begin{aligned}
\liminf _{L \rightarrow \infty} \frac{-\ln \mathrm{P}_{\mathrm{e}}\left(R_{L+3 \sqrt{L}}, L+3 \sqrt{L}\right)}{(L+3 \sqrt{L}) \rho_{L+3 \sqrt{L}}} & =\liminf _{L \rightarrow \infty} \frac{-\ln \mathrm{P}_{\mathrm{e}}\left(R_{L+3 \sqrt{L}}, L+3 \sqrt{L}\right)}{L \rho_{L}^{\prime}} \\
& =\liminf _{L \rightarrow \infty} \frac{-\ln \mathrm{P}_{\mathrm{e}}^{\prime}\left(R_{L}, L\right)}{L \rho_{L}^{\prime}} \geq \frac{B}{C} .
\end{aligned}
$$

By choosing $L$ such that $L+3 \sqrt{L}=N$, this is equivalent to assertion of the existence of an $\left(\exp \left(N\left(C-\rho_{N}\right)\right), N\right)$-VLF code such that

$$
\liminf _{N \rightarrow \infty} \frac{-\ln \mathrm{P}_{\mathrm{e}}\left(R_{N}, N\right)}{N \rho_{N}} \geq \frac{B}{C} .
$$

This concludes the proof for the achievability part of Theorem 1.

\section{Converse Proof}

To prove the converse, we use similar proof arguments as in Burnashev's paper [4] together with Lemmas 9 and 10 below. We first state some of Burnashev's lemmas that are used in our converse proof.

Lemma 5. Under the condition that $\mathbb{P}(\tau<\infty)=1$, the following Fano-type inequality holds

$$
\mathbb{E}\left[\mathcal{H}\left(W \mid Y^{\tau}\right)\right] \leq h\left(\mathrm{P}_{\mathrm{e}}\left(R_{N}, N\right)\right)+\mathrm{P}_{\mathrm{e}}\left(R_{N}, N\right) \ln (M-1) .
$$

Lemma 6. For any $n \geq 0$ the following inequality holds almost surely

$$
\mathbb{E}\left[\mathcal{H}\left(W \mid Y^{n}\right)-\mathcal{H}\left(W \mid Y^{n+1}\right) \mid Y^{n}\right] \leq C .
$$

Lemma 7. For any $n \geq 0$ the following inequality holds almost surely

$$
\mathbb{E}\left[\ln \mathcal{H}\left(W \mid Y^{n}\right)-\ln \mathcal{H}\left(W \mid Y^{n+1}\right) \mid Y^{n}\right] \leq B .
$$

Proof of Lemma 7: Please see Appendix E in which we provide a self-contained proof by combining ideas in [4, Lemma 3] and Burnashev's sequential hypothesis testing paper [9, Lemma 3].

Remark 4. Some remarks concerning Lemma 7 are in order.

- This lemma is less strict than (i.e., a generalization of) the original version in [4, Lemma 3]. We have removed the boundedness assumption, i.e., that $\mathcal{H}\left(W \mid Y^{n}\right) \leq \mathcal{H}^{*}\left(P_{Y \mid X}\right)$ where $\mathcal{H}^{*}\left(P_{Y \mid X}\right)$ is some function of the DMC $P_{Y \mid X}$ in [4, Lemma 3].

- Without the boundedness assumption that $\mathcal{H}\left(W \mid Y^{n}\right) \leq \mathcal{H}^{*}\left(P_{Y \mid X}\right)$, the proof of [4, Theorem 1] which leads to the error exponent result in (1) can be simplified thanks to a simpler construction of an appropriate submartingale.

Lemma 8. The following inequality holds almost surely

$$
\mathbb{E}\left[\left(\ln \mathcal{H}\left(W \mid Y^{n}\right)-\ln \mathcal{H}\left(W \mid Y^{n+1}\right)\right)_{\vartheta} \mid Y^{n}\right] \leq \varphi(\vartheta)
$$

where $\varphi(\vartheta)=(\ln T)_{\vartheta}$ and $T$ is defined in (13). Under the condition $B<\infty, \varphi(\vartheta)=0$ for $\vartheta$ sufficiently large.

Now, we present two new lemmas that are useful to analyze the moderate deviations regime. Lemma 9 is important to deal with the case in which $R_{N}$ is strictly larger than $C-\rho_{N}$; this is allowed by Definition 2. Lemma 10 is used to show an important result that for any $\left(\exp \left(N\left(C-\rho_{N}\right)\right), N\right)$-VLF code, (161) in the proof of the converse holds.

${ }^{3}$ Note that this code has the same average error probability as the $\left(\exp \left(L\left(C-\rho_{L}^{\prime}\right)\right), L+3 \sqrt{L}\right)$-VLF code, i.e., $\mathrm{P}_{\mathrm{e}}\left(R_{L+3 \sqrt{L}}, L+3 \sqrt{L}\right)=\mathrm{P}_{\mathrm{e}}^{\prime}\left(R_{L}, L\right)$. 
Lemma 9. Let $\left\{x_{n}\right\}_{n \in \mathbb{R}_{+}}$and $\left\{y_{n}\right\}_{n \in \mathbb{R}_{+}}$be two families of non-negative numbers such that

$$
\limsup _{k \rightarrow \infty} x_{n_{k}} \geq \limsup _{k \rightarrow \infty} y_{n_{k}} \geq 0
$$

for any pair of subsequences $\left(\left\{x_{n_{k}}\right\}_{k=1}^{\infty},\left\{y_{n_{k}}\right\}_{k=1}^{\infty}\right)$ of the original families $\left(\left\{x_{n}\right\}_{n \in \mathbb{R}_{+}},\left\{y_{n}\right\}_{n \in \mathbb{R}_{+}}\right)$. In addition, assume that

$$
\liminf _{n \rightarrow \infty} x_{n}=0 .
$$

Then, we have

$$
\liminf _{n \rightarrow \infty} y_{n}=0
$$

Proof of Lemma 9: Please see Appendix F.

Lemma 10. Assume that $\left\{\phi_{N}\right\}_{N \in \mathbb{R}_{+}}$is a family of real numbers in the open interval $(0,1)$ satisfying

$$
\begin{aligned}
- & \frac{\ln \phi_{N}}{N \rho_{N}}-\frac{\ln \left(N C-N \rho_{N}-\ln \phi_{N}\right)}{N \rho_{N}} \\
& \leq \frac{B}{C N \rho_{N}}+\frac{B \phi_{N}}{\rho_{N}}-\frac{B \phi_{N}}{C}+\frac{B}{C}+\frac{O(1)}{N \rho_{N}},
\end{aligned}
$$

where $O(1)$ is a bounded constant as $N \rightarrow \infty$. Here, $\left\{\rho_{N}\right\}_{N=1}^{\infty}$ is a family of real positive numbers satisfying (6) and (7). Then, we have

$$
\limsup _{N \rightarrow \infty}-\frac{\ln \phi_{N}}{N}<\infty .
$$

Proof of Lemma 10: Please see Appendix G.

We are now ready to present the proof of the converse.

Proposition 2 (Converse of Theorem 1). Under the conditions of Theorem 1,

$$
E^{*} \leq \frac{B}{C}
$$

The main contribution of the proof of Proposition 2 is to supplement new mathematical analyses that are amenable to the moderate deviations setting. In the following proof, all the steps from (151) to (171) are different from Burnashev's work [4].

Remark 5. We remark that Berlin et al. [10] provided a simple and elegant converse proof of (1) by emulating the two-phase achievability proof in Yamamoto and Itoh's paper [8]. The approach in [10] may possibly be also used to obtain an upper bound for the optimal moderate deviations constant. However, an optimal way to choose the parameter $\delta$ in $[10$, Theorem 1] is not obvious (at least to the authors). This is because of the presence of the multiplicative term, i.e., $\left(1-\delta-\mathrm{P}_{\mathrm{e}} / \delta\right)$, right before $(\ln M) / C$ and the additive term $(\ln (\lambda \delta)-\ln 4) / B$ in [10, Eqn. (18)]. In addition, there are some limiting statements that have to be modified and it is not clear how to do so in a rigorous and optimal manner (to prove a tight upper bound on $E^{*}$ ).

Proof of Proposition 2: For $R_{N} \geq C-\rho_{N}$, we first realize that if there exists an $\left(\exp \left(N R_{N}\right), N\right)$-VLF code with an average error probability $\mathrm{P}_{\mathrm{e}}\left(R_{N}, N\right)$, we can find a $\left(\exp \left(N\left(C-\rho_{N}\right)\right), N\right)$-VLF code with an average error probability $\mathrm{P}_{\mathrm{e}}\left(C-\rho_{N}, N\right)$ satisfying $\mathrm{P}_{\mathrm{e}}\left(C-\rho_{N}, N\right) \leq \mathrm{P}_{\mathrm{e}}\left(R_{N}, N\right)$ by removing $\exp \left(N R_{N}\right)-\exp \left(N\left(C-\rho_{N}\right)\right)$ messages with the highest conditional error probabilities. This means that $\inf _{N \in \mathcal{A}} \mathrm{P}_{\mathrm{e}}\left(R_{N}, N\right) \geq \inf _{N \in \mathcal{A}} \mathrm{P}_{\mathrm{e}}\left(C-\rho_{N}, N\right)$ for any $\mathcal{A} \subset \mathbb{R}_{+}$. Hence,

$$
\limsup _{k \rightarrow \infty}-\frac{\ln \mathrm{P}_{\mathrm{e}}\left(C-\rho_{N_{k}}, N_{k}\right)}{N_{k} \rho_{N_{k}}} \geq \limsup _{k \rightarrow \infty}-\frac{\ln \mathrm{P}_{\mathrm{e}}\left(R_{N_{k}}, N_{k}\right)}{N_{k} \rho_{N_{k}}},
$$

for any increasing subsequence of positive numbers $\left\{N_{k}\right\}_{k=1}^{\infty}$. To show (117) we consider two cases:

- Case 1: There exists a family of $\left(\exp \left(N\left(C-\rho_{N}\right)\right), N\right)-\mathrm{VLF}$ codes such that

$$
\liminf _{N \rightarrow \infty}-\frac{\ln \mathrm{P}_{\mathrm{e}}\left(C-\rho_{N}, N\right)}{N \rho_{N}}=0 .
$$

It follows that (117) trivially holds since from (118), (119), and Lemma 9 (with $\phi_{N}=\mathrm{P}_{\mathrm{e}}\left(R_{N}, N\right)$ ) we must have

$$
\liminf _{N \rightarrow \infty}-\frac{\ln \mathrm{P}_{\mathrm{e}}\left(R_{N}, N\right)}{N \rho_{N}}=0,
$$

which leads to

$$
E^{*} \leq \liminf _{N \rightarrow \infty}-\frac{\ln \mathrm{P}_{\mathrm{e}}\left(R_{N}, N\right)}{N \rho_{N}}=0 \leq \frac{B}{C} .
$$


- Case 2: For all families of $\left(\exp \left(N\left(C-\rho_{N}\right)\right), N\right)$-VLF codes, the following holds

$$
\alpha=\liminf _{N \rightarrow \infty}-\frac{\ln \mathrm{P}_{\mathrm{e}}\left(C-\rho_{N}, N\right)}{N \rho_{N}}>0 .
$$

For this case, we first prove that

$$
\limsup _{N \rightarrow \infty}-\frac{\ln \mathrm{P}_{\mathrm{e}}\left(C-\rho_{N}, N\right)}{N \rho_{N}} \leq \frac{B}{C}
$$

for any $\left(\exp \left(N\left(C-\rho_{N}\right)\right), N\right)$-VLF codes. Next, we extend the analysis to $\left(\exp \left(N R_{N}\right), N\right)$-VLF codes for $R_{N} \geq C-\rho_{N}$.

To prove (123) for all $\left(\exp \left(N\left(C-\rho_{N}\right)\right), N\right)$-VLF codes satisfying (122), we use the same converse proof techniques as Burnashev [4] with some augmented arguments to account for the fact that the code is in the moderate deviations regime. Here, a combination of [4] and [9] makes the proof that the sequence $\zeta_{n}$ (to be defined in (125) in the following) is a submartingale simpler. For completeness, we provide the entire proof for this case.

To begin with, we show the following inequality

$$
\begin{aligned}
- & \frac{\ln \mathrm{P}_{\mathrm{e}}\left(C-\rho_{N}, N\right)}{N \rho_{N}}-\frac{\ln \left(N C-N \rho_{N}-\ln \mathrm{P}_{\mathrm{e}}\left(C-\rho_{N}, N\right)\right)}{N \rho_{N}} \\
& \leq \frac{B}{C N \rho_{N}}+\frac{B \mathrm{P}_{\mathrm{e}}\left(C-\rho_{N}, N\right)}{\rho_{N}}-\frac{B \mathrm{P}_{\mathrm{e}}\left(C-\rho_{N}, N\right)}{C}+\frac{B}{C}+\frac{O(1)}{N \rho_{N}} .
\end{aligned}
$$

Here, $O(1)$ is a bounded constant as $N \rightarrow \infty$.

It is enough to show that (124) holds for $N<\infty$, i.e., $\mathbb{E}(\tau)<\infty$. We also assume that $B<\infty$, otherwise (123) obviously holds. Now, as in Burnashev's arguments [9], we consider a random sequence

$$
\zeta_{n}=\left\{\begin{array}{ll}
C^{-1} \mathcal{H}\left(W \mid Y^{n}\right)+n, & \text { if } \quad \mathcal{H}\left(W \mid Y^{n}\right) \geq A, \\
B^{-1} \ln \mathcal{H}\left(W \mid Y^{n}\right)+b+n, & \text { if } \quad \mathcal{H}\left(W \mid Y^{n}\right) \leq A
\end{array} .\right.
$$

where $A$ is the largest positive root of the following equation in $x$ :

$$
\frac{x}{C}=\frac{\ln x}{B}+b .
$$

For $b$ sufficiently large, we will show that the sequence $\zeta_{n}$ forms a submartingle with respect to the filtration $\left\{\sigma\left(Y^{n}\right)\right\}_{n=0}^{\infty}$. Note that when $b$ sufficiently large, (126) can be shown to have two distinct positive roots $a, A$ and that $A / a$ can be make arbitrarily large by increasing $b[4$, pp. 256].

Indeed, first we suppose that $\mathcal{H}\left(W \mid Y^{n}\right) \leq A$. Then, we obtain

$$
\begin{array}{rl}
\mathbb{E}\left[\zeta_{n}-\zeta_{n+1} \mid Y^{n}\right]=-1 & \mathbb{E}\left[B^{-1} \ln \mathcal{H}\left(W \mid Y^{n}\right)+b-\left(B^{-1} \ln \mathcal{H}\left(W \mid Y^{n+1}\right)+b\right) \mathbf{1}\left\{\mathcal{H}\left(W \mid Y^{n+1}\right) \leq A\right\}\right. \\
& \left.-C^{-1} \mathcal{H}\left(W \mid Y^{n+1}\right) \mathbf{1}\left\{\mathcal{H}\left(W \mid Y^{n+1}\right)>A\right\} \mid Y^{n}\right] \\
\leq-1 & +B^{-1} \mathbb{E}\left[\ln \mathcal{H}\left(W \mid Y^{n}\right)-\ln \mathcal{H}\left(W \mid Y^{n+1}\right) \mid Y^{n}\right] \\
\leq & -1+B^{-1} \times B=0 .
\end{array}
$$

Here, (128) follows from the fact that $x / C \geq(\ln x) / B+b$ for $x \geq A[4$, pp. 256] and (129) follows from Lemma 7.

Now, suppose that $\mathcal{H}\left(W \mid Y^{n}\right)>A$. Let $a$ be the smaller of the two positive roots of (126). Then, for $b$ sufficiently large,

$$
\begin{aligned}
\mathbb{E} & {\left[\zeta_{n}-\zeta_{n+1} \mid Y^{n}\right] } \\
= & -1+C^{-1} \mathbb{E}\left[\mathcal{H}\left(W \mid Y^{n}\right)-\mathcal{H}\left(W \mid Y^{n+1}\right) \mid Y^{n}\right] \\
& +\mathbb{E}\left[\left(C^{-1} \mathcal{H}\left(W \mid Y^{n+1}\right)-B^{-1} \ln \mathcal{H}\left(W \mid Y^{n+1}\right)-b\right) \mathbf{1}\left\{\mathcal{H}\left(W \mid Y^{n+1}\right) \leq A\right\} \mid Y^{n}\right] \\
\leq & \mathbb{E}\left[\left(C^{-1} \mathcal{H}\left(W \mid Y^{n+1}\right)-B^{-1} \ln \mathcal{H}\left(W \mid Y^{n+1}\right)-b\right) \mathbf{1}\left\{\mathcal{H}\left(W \mid Y^{n+1}\right) \leq A\right\} \mid Y^{n}\right] \\
= & \mathbb{E}\left[\left(C^{-1} \mathcal{H}\left(W \mid Y^{n+1}\right)-B^{-1} \ln \mathcal{H}\left(W \mid Y^{n+1}\right)-b\right) \mathbf{1}\left\{\mathcal{H}\left(W \mid Y^{n+1}\right) \leq a\right\} \mid Y^{n}\right] \\
& +\mathbb{E}\left[\left(C^{-1} \mathcal{H}\left(W \mid Y^{n+1}\right)-B^{-1} \ln \mathcal{H}\left(W \mid Y^{n+1}\right)-b\right) \mathbf{1}\left\{a<\mathcal{H}\left(W \mid Y^{n+1}\right) \leq A\right\} \mid Y^{n}\right] \\
\leq & \mathbb{E}\left[\left(C^{-1} \mathcal{H}\left(W \mid Y^{n+1}\right)-B^{-1} \ln \mathcal{H}\left(W \mid Y^{n+1}\right)-b\right) \mathbf{1}\left\{\mathcal{H}\left(W \mid Y^{n+1}\right) \leq a\right\} \mid Y^{n}\right] \\
\leq & B^{-1} \mathbb{E}\left[\left(\ln \mathcal{H}\left(W \mid Y^{n}\right)-\ln \mathcal{H}\left(W \mid Y^{n+1}\right)\right) \mathbf{1}\left\{\mathcal{H}\left(W \mid Y^{n+1}\right) \leq a\right\} \mid Y^{n}\right] \\
\leq & B^{-1} \mathbb{E}\left[\left(\ln \mathcal{H}\left(W \mid Y^{n}\right)-\ln \mathcal{H}\left(W \mid Y^{n+1}\right)\right) \mathbf{1}\left\{\ln \mathcal{H}\left(W \mid Y^{n}\right)-\ln \mathcal{H}\left(W \mid Y^{n+1}\right)>\ln \left(\frac{A}{a}\right)\right\} \mid Y^{n}\right] \\
= & B^{-1} \mathbb{E}\left[\left(\ln \mathcal{H}\left(W \mid Y^{n}\right)-\ln \mathcal{H}\left(W \mid Y^{n+1}\right)\right)_{\ln \left(\frac{A}{a}\right)} \mid Y^{n}\right] \\
\leq & B^{-1} \varphi\left(\ln \left(\frac{A}{a}\right)\right) \\
= & 0 .
\end{aligned}
$$


In the above chain of inequalities, (130) follows from (125), (131) follows from Lemma 6, (133) follows from the fact that $C^{-1} \mathcal{H}\left(W \mid Y^{n+1}\right) \leq B^{-1} \ln \mathcal{H}\left(W \mid Y^{n+1}\right)+b$ if $a<\mathcal{H}\left(W \mid Y^{n+1}\right) \leq A$, (134) follows from the fact that if $\mathcal{H}\left(W \mid Y^{n+1}\right) \leq a$ and $\mathcal{H}\left(W \mid Y^{n}\right)>A$ we have $C^{-1} \mathcal{H}\left(W \mid Y^{n+1}\right)-b \leq C^{-1} a-b=B^{-1} \ln a \leq B^{-1} \ln A \leq B^{-1} \ln \mathcal{H}\left(W \mid Y^{n}\right)$, (135) follows from the assumption that $\mathcal{H}\left(W \mid Y^{n}\right)>A$, (136) follows from usage of the notation $(x)_{a}=x \mathbf{1}\{x \geq a\}$, and (137) and (138) follow from Lemma 8 and the fact that $A / a$ can be made arbitrarily large by increasing $b$. Inequalities (138) and (129) confirm that $\zeta_{n}$ forms a submartingale with respect to $\left\{\sigma\left(Y^{n}\right)\right\}_{n=0}^{\infty}$.

Now, since we know that

$$
\zeta_{0}=\mathbb{E}\left[\zeta_{0}\right] \leq \mathbb{E}\left[\zeta_{n \wedge \tau}\right] \leq \limsup _{n \rightarrow \infty} \mathbb{E}\left[\zeta_{n \wedge \tau}\right],
$$

it follows that for $N$ sufficiently large we have

$$
\begin{aligned}
C^{-1}\left(N C-N \rho_{N}\right)= & \zeta_{0}^{(1)} \\
\leq & \limsup _{n \rightarrow \infty} \mathbb{E}\left[\zeta_{n \wedge \tau}\right] \\
\leq & C^{-1} \limsup _{n \rightarrow \infty} \mathbb{E}\left[\mathcal{H}\left(W \mid Y^{\tau \wedge n}\right) \mathbf{1}\left\{\mathcal{H}\left(W \mid Y^{\tau \wedge n}\right) \geq A\right\}\right] \\
& +\limsup _{n \rightarrow \infty} \mathbb{E}[\tau \wedge n]+\limsup _{n \rightarrow \infty} B^{-1} \mathbb{E}\left[\ln \mathcal{H}\left(W \mid Y^{\tau \wedge n}\right) \mathbf{1}\left\{\mathcal{H}\left(W \mid Y^{\tau \wedge n}\right) \leq A\right\}\right]+b \\
\leq & C^{-1} \limsup _{n \rightarrow \infty} \mathbb{E}\left[\mathcal{H}\left(W \mid Y^{\tau \wedge n}\right)\right] \\
& +\limsup _{n \rightarrow \infty} \mathbb{E}[\tau \wedge n]+\limsup _{n \rightarrow \infty} B^{-1} \mathbb{E}\left[\ln \mathcal{H}\left(W \mid Y^{\tau \wedge n}\right) \mathbf{1}\left\{\mathcal{H}\left(W \mid Y^{\tau \wedge n}\right) \leq A\right\}\right]+b \\
\leq & C^{-1} \limsup _{n \rightarrow \infty} \mathbb{E}\left[\mathcal{H}\left(W \mid Y^{\tau \wedge n}\right)\right]+\limsup _{n \rightarrow \infty} \mathbb{E}[\tau \wedge n]+\limsup _{n \rightarrow \infty} B^{-1} \ln \mathbb{E}\left[\mathcal{H}\left(W \mid Y^{\tau \wedge n}\right)\right]+b \\
= & C^{-1} \mathbb{E}\left[\mathcal{H}\left(W \mid Y^{\tau}\right)\right]+\mathbb{E}[\tau]+B^{-1} \mathbb{E}\left[\ln \mathcal{H}\left(W \mid Y^{\tau}\right)\right] \\
\leq & C^{-1}\left[1+\mathrm{P}_{\mathrm{e}}\left(C-\rho_{N}, N\right)\left(N C-N \rho_{N}\right)\right]+\mathbb{E}[\tau]+B^{-1} \ln \left[h\left(\mathrm{P}_{\mathrm{e}}\left(C-\rho_{N}, N\right)\right)\right. \\
& \left.+\mathrm{P}_{\mathrm{e}}\left(C-\rho_{N}, N\right)\left(N C-N \rho_{N}\right)\right]+b \\
= & C^{-1}\left[1+\mathrm{P}_{\mathrm{e}}\left(C-\rho_{N}, N\right)\left(N C-N \rho_{N}\right)\right]+\mathbb{E}[\tau]+B^{-1} \ln \left[-\mathrm{P}_{\mathrm{e}}\left(C-\rho_{N}, N\right) \ln \mathrm{P}_{\mathrm{e}}\left(C-\rho_{N}, N\right)\right. \\
& \left.-\left(1-\mathrm{P}_{\mathrm{e}}\left(C-\rho_{N}, N\right)\right) \ln \left(1-\mathrm{P}_{\mathrm{e}}\left(C-\rho_{N}, N\right)\right)+\mathrm{P}_{\mathrm{e}}\left(C-\rho_{N}, N\right)\left(N C-N \rho_{N}\right)\right]+b \\
\leq & C^{-1}\left[1+\mathrm{P}_{\mathrm{e}}\left(C-\rho_{N}, N\right)\left(N C-N \rho_{N}\right)\right]+\mathbb{E}[\tau]+B^{-1} \ln \left[-\mathrm{P}_{\mathrm{e}}\left(C-\rho_{N}, N\right) \ln \mathrm{P}_{\mathrm{e}}\left(C-\rho_{N}, N\right)\right. \\
& \left.+\frac{1}{e}+\mathrm{P}_{\mathrm{e}}\left(C-\rho_{N}, N\right)\left(N C-N \rho_{N}\right)\right]+b \\
= & C^{-1}\left[1+\mathrm{P}_{\mathrm{e}}\left(C-\rho_{N}, N\right)\left(N C-N \rho_{N}\right)\right]+\mathbb{E}[\tau]+B^{-1} \ln \left[-\mathrm{P}_{\mathrm{e}}\left(C-\rho_{N}, N\right) \ln \mathrm{P}_{\mathrm{e}}\left(C-\rho_{N}, N\right)\right. \\
& \left.+\mathrm{P}_{\mathrm{e}}\left(C-\rho_{N}, N\right)\left(N C-N \rho_{N}\right)\right]+O(1) \\
\leq & C^{-1}\left[1+\mathrm{P}_{\mathrm{e}}\left(C-\rho_{N}, N\right)\left(N C-N \rho_{N}\right)\right]+N+B^{-1} \ln \mathrm{P}_{\mathrm{e}}\left(C-\rho_{N}, N\right) \\
& +B^{-1} \ln \left(N C-N \rho_{N}-\ln \mathrm{P}_{\mathrm{e}}\left(C-\rho_{N}, N\right)\right)+O(1) .
\end{aligned}
$$

Here, (140) follows from (125) and $\mathcal{H}\left(W \mid Y^{0}\right)=H(W)=N C-N \rho_{N}$, (141) follows from (139), (142) follows from (125) and (139), (144) follows from the fact that for any random variable $U, \mathbb{E}[(\ln U) \mathbf{1}\{U \leq u\}] \leq \ln \mathbb{E}(U)$ for all $u \geq 1$ (which is assured by taking $b$ sufficiently large so $A$ eventually becomes larger than 1), (146) follows from Lemma 5 for the case that $R_{N}=C-\rho_{N}$, (148) follows from the fact that $-x \ln x \leq 1 / e$ for $0 \leq x \leq 1$, (149) follows from the fact that $b<\infty$, and (150) follows from (5).

It follows from (150) that

$$
\begin{aligned}
-B^{-1} \ln \mathrm{P}_{\mathrm{e}}\left(C-\rho_{N}, N\right) \leq & C^{-1}\left[1+\mathrm{P}_{\mathrm{e}}\left(C-\rho_{N}, N\right) N\left(C-\rho_{N}\right)\right]+C^{-1} N \rho_{N} \\
& +B^{-1} \ln \left(N\left(C-\rho_{N}\right)-\ln \mathrm{P}_{\mathrm{e}}\left(C-\rho_{N}, N\right)\right)+O(1) .
\end{aligned}
$$

Hence, we have

$$
\begin{aligned}
- & \frac{\ln \mathrm{P}_{\mathrm{e}}\left(C-\rho_{N}, N\right)}{N \rho_{N}}-\frac{\ln \left(N C-N \rho_{N}-\ln \mathrm{P}_{\mathrm{e}}\left(C-\rho_{N}, N\right)\right)}{N \rho_{N}} \\
& \leq \frac{B}{C N \rho_{N}}+\frac{B \mathrm{P}_{\mathrm{e}}\left(C-\rho_{N}, N\right)}{\rho_{N}}-\frac{B \mathrm{P}_{\mathrm{e}}\left(C-\rho_{N}, N\right)}{C}+\frac{B}{C}+\frac{O(1)}{N \rho_{N}},
\end{aligned}
$$

and (124) is shown.

Now, from the assumption in (122), we have

$$
-\frac{\ln \mathrm{P}_{\mathrm{e}}\left(C-\rho_{N}, N\right)}{N \rho_{N}} \geq \frac{\alpha}{2}
$$


for $N$ sufficiently large. It follows that

$$
\mathrm{P}_{\mathrm{e}}\left(C-\rho_{N}, N\right) \leq \exp \left(-\frac{\alpha}{2} N \rho_{N}\right)
$$

Hence, we have

$$
0 \leq \limsup _{N \rightarrow \infty} \frac{\mathrm{P}_{\mathrm{e}}\left(C-\rho_{N}, N\right)}{\rho_{N}} \leq \limsup _{N \rightarrow \infty} \frac{\exp \left(-\frac{\alpha}{2} N \rho_{N}\right)}{\rho_{N}}
$$

Now, we note that for any $\beta>0, g_{\beta}(x)=\frac{1}{x} \exp (-\beta x)$ is a decreasing function in $x \in(0, \infty)$. Moreover, since $\sqrt{N} \rho_{N} \rightarrow \infty$ as $N \rightarrow \infty$, there exists $N_{0}$ sufficiently large such that $\sqrt{N} \rho_{N} \geq 1$ for all $N \geq N_{0}$. It follows that for all $N \geq N_{0}$ we have

$$
\frac{\exp \left(-\frac{\alpha}{2} \sqrt{N}\left(\sqrt{N} \rho_{N}\right)\right)}{\sqrt{N} \rho_{N}}=g_{\frac{\alpha}{2} \sqrt{N}}\left(\sqrt{N} \rho_{N}\right) \leq g_{\frac{\alpha}{2} \sqrt{N}}(1)=\exp \left(-\frac{\alpha}{2} \sqrt{N}\right)
$$

Hence, we obtain

$$
0 \leq \liminf _{N \rightarrow \infty} \frac{\exp \left(-\frac{\alpha}{2} N \rho_{N}\right)}{\rho_{N}} \leq \limsup _{N \rightarrow \infty} \frac{\exp \left(-\frac{\alpha}{2} N \rho_{N}\right)}{\rho_{N}} \leq \limsup _{N \rightarrow \infty}\left[\exp \left(-\frac{\alpha}{2} \sqrt{N}\right)\right] \sqrt{N}=0 .
$$

From (155) and (157) we obtain

$$
\lim _{N \rightarrow \infty} \frac{\mathrm{P}_{\mathrm{e}}\left(C-\rho_{N}, N\right)}{\rho_{N}}=\lim _{N \rightarrow \infty}\left[\exp \left(-\frac{\alpha}{2} \sqrt{N}\right)\right] \sqrt{N}=0 .
$$

By taking lim supremum both sides of (124) and using (158), we have

$$
\begin{aligned}
\frac{B}{C} & \geq \limsup _{N \rightarrow \infty}\left[-\frac{\ln \mathrm{P}_{\mathrm{e}}\left(C-\rho_{N}, N\right)}{N \rho_{N}}\right]+\liminf _{N \rightarrow \infty}\left[-\frac{\left.\ln \left(N C-N \rho_{N}\right)-\ln \mathrm{P}_{\mathrm{e}}\left(C-\rho_{N}, N\right)\right)}{N \rho_{N}}\right] \\
& =\limsup _{N \rightarrow \infty}\left[-\frac{\ln \mathrm{P}_{\mathrm{e}}\left(C-\rho_{N}, N\right)}{N \rho_{N}}\right]-\limsup _{N \rightarrow \infty}\left[\frac{\left.\ln \left(N C-N \rho_{N}\right)-\ln \mathrm{P}_{\mathrm{e}}\left(C-\rho_{N}, N\right)\right)}{N \rho_{N}}\right] .
\end{aligned}
$$

Next, we will show that

$$
\lim _{N \rightarrow \infty}\left[\frac{\left.\ln \left(N C-N \rho_{N}\right)-\ln \mathrm{P}_{\mathrm{e}}\left(C-\rho_{N}, N\right)\right)}{N \rho_{N}}\right]=0 .
$$

and so the second term in (160) is zero. Observe that

$$
\begin{aligned}
\frac{\ln \left(N C-N \rho_{N}-\ln \mathrm{P}_{\mathrm{e}}\left(C-\rho_{N}, N\right)\right)}{N \rho_{N}} & =\frac{\ln \left(N C-N \rho_{N}-\ln \mathrm{P}_{\mathrm{e}}\left(C-\rho_{N}, N\right)\right)}{N \rho_{N}} \\
& \geq \frac{\ln \left(C-\rho_{N}\right)}{\sqrt{N}\left(\sqrt{N} \rho_{N}\right)}+\frac{\ln N}{\sqrt{N}} \cdot \frac{1}{\sqrt{N} \rho_{N}}
\end{aligned}
$$

Here, (163) follows from the fact that $0<\mathrm{P}_{\mathrm{e}}\left(C-\rho_{N}, N\right) \leq 1$. It follows that

$$
\liminf _{N \rightarrow \infty} \frac{\ln \left(N C-N \rho_{N}-\ln \mathrm{P}_{\mathrm{e}}\left(C-\rho_{N}, N\right)\right)}{N \rho_{N}} \geq \liminf _{N \rightarrow \infty} \frac{\ln \left(C-\rho_{N}\right)}{\sqrt{N}\left(\sqrt{N} \rho_{N}\right)}+\frac{\ln N}{\sqrt{N}} \cdot \frac{1}{\sqrt{N} \rho_{N}}=0
$$

Now, from (124) and Lemma 10, we have

$$
\limsup _{N \rightarrow \infty}-\frac{\ln \mathrm{P}_{\mathrm{e}}\left(C-\rho_{N}, N\right)}{N}<\infty
$$

It follows from (165) we have for $N$ sufficiently large that

$$
-\frac{\ln \mathrm{P}_{\mathrm{e}}\left(C-\rho_{N}, N\right)}{N} \leq \nu
$$

for some constant $\nu \in(0,+\infty)$. Hence, we have

It follows that

$$
\begin{aligned}
\frac{\ln \left(N C-N \rho_{N}-\ln \mathrm{P}_{\mathrm{e}}\left(C-\rho_{N}, N\right)\right)}{N \rho_{N}} & =\frac{\ln \left(N C-N \rho_{N}-\ln \mathrm{P}_{\mathrm{e}}\left(C-\rho_{N}, N\right)\right)}{N \rho_{N}} \\
& \leq \frac{\ln \left(C-\rho_{N}+\nu\right)}{\sqrt{N}\left(\sqrt{N} \rho_{N}\right)}+\frac{\ln N}{\sqrt{N}} \cdot \frac{1}{\sqrt{N} \rho_{N}}
\end{aligned}
$$

$$
\begin{aligned}
\limsup _{N \rightarrow \infty} \frac{\ln \left(N C-N \rho_{N}-\ln \mathrm{P}_{\mathrm{e}}\left(C-\rho_{N}, N\right)\right)}{N \rho_{N}} & \leq \limsup _{N \rightarrow \infty} \frac{\ln \left(C-\rho_{N}+\nu\right)}{\sqrt{N}\left(\sqrt{N} \rho_{N}\right)}+\frac{\ln N}{\sqrt{N}} \cdot \frac{1}{\sqrt{N} \rho_{N}} \\
& =0 .
\end{aligned}
$$


Combining (164) and (170), we obtain (161). From (160) and (161), we obtain (123) as desired.

Finally, for any $\left(\exp \left(N R_{N}\right), N\right)$-VLF codes, by combining (9), (118), and (123) we have

$$
E^{*} \leq \liminf _{N \rightarrow \infty}\left[-\frac{\ln \mathrm{P}_{\mathrm{e}}\left(R_{N}, N\right)}{N \rho_{N}}\right] \leq \limsup _{N \rightarrow \infty}\left[-\frac{\ln \mathrm{P}_{\mathrm{e}}\left(R_{N}, N\right)}{N \rho_{N}}\right] \leq \limsup _{N \rightarrow \infty}\left[-\frac{\ln \mathrm{P}_{\mathrm{e}}\left(C-\rho_{N}, N\right)}{N \rho_{N}}\right] \leq \frac{B}{C},
$$

concluding the proof for the converse.

\section{APPENDIX A}

PRELIMINARIES FOR THE PROOF OF LEMmas 1, 2, AND 3

To prove Lemma 1, 2, and 3, we first state and prove some useful preliminary definitions and lemmas.

Definition 4. Let $\left\{U_{n}\right\}_{n=1}^{\infty}$ be a sequence of random variables. The sequence $\left\{U_{n}\right\}_{n=1}^{\infty}$ is called $*$-submixing sequence adapted to a filtration $\left\{\mathcal{F}_{n}\right\}_{n=1}^{\infty}$ if $U_{n} \in \mathcal{F}_{n}$ and that there exists a positive number $N$ and a non-negative function $f$ defined on the integers $n \geq N$ such that $f(n) \rightarrow 0$ as $n \rightarrow \infty$ and for all $n \geq N, m \geq 1$,

$$
\left|\mathbb{E}\left(U_{n+m} \mid \mathcal{F}_{m}\right)-\mathbb{E}\left(U_{n+m}\right)\right| \leq f(n) \mathbb{E}\left|U_{n+m}\right| .
$$

Lemma 11. [29, Theorem 2.18] Let $\left\{W_{i}\right\}_{i=1}^{\infty}$ be a sequence of random variables such that $\left\{S_{n}=\sum_{i=1}^{n} W_{i}, \mathcal{F}_{n}\right\}_{n=1}^{\infty}$ is a martingale, and let $\left\{V_{n}\right\}_{n=1}^{\infty}$ be a non-decreasing sequence of positive random variables such that $V_{n} \in \mathcal{F}_{n-1}$ for each n. Fix $1 \leq p \leq 2$. Then

$$
\lim _{n \rightarrow \infty} V_{n}^{-1} S_{n}=0, \quad \text { a.s. }
$$

on the event

$$
\left\{\lim _{n \rightarrow \infty} V_{n}=\infty, \sum_{i=1}^{\infty} V_{i}^{-p} \mathbb{E}\left[\left|W_{i}\right|^{p} \mid \mathcal{F}_{i-1}\right]<\infty\right\} .
$$

Lemma 12. Let $\left\{U_{n}\right\}_{n=1}^{\infty}$ be a $*$-submixing sequence adapted to a filtration $\left\{\mathcal{F}_{n}\right\}_{n=1}^{\infty}$ such that $\mathbb{E}\left(U_{n}\right)=0$ and $\mathbb{E}\left(U_{n}^{2}\right)<$ $\infty, n \geq 1$. Suppose that $\sum_{n=1}^{\infty} b_{n}^{-2} \mathbb{E}\left(U_{n}^{2}\right)<\infty$, and $\sup _{n} b_{n}^{-1} \sum_{i=1}^{n} \mathbb{E}\left|U_{i}\right|<\infty$, where $\left\{b_{n}\right\}_{n=1}^{\infty}$ is a sequence of positive constants increasing to $\infty$. Then,

$$
b_{n}^{-1} \sum_{i=1}^{n} U_{i} \rightarrow 0, \quad \text { a.s. }
$$

Remark 6. Some remarks concerning Lemma 12 are in order.

- This lemma is a generalization of [29, Theorem 2.20], which considered the case where $\mathcal{F}_{n}=\sigma\left(U_{1}^{n}\right)$ and that $\left\{U_{n}\right\}_{n=1}^{\infty}$ is a $*$-mixing sequence in the sense that for any $B \in \mathcal{F}_{\infty}=\lim _{n \rightarrow \infty} \mathcal{F}_{n}$,

$$
\left|\mathbb{P}\left(B \mid \mathcal{F}_{n}\right)-\mathbb{P}(B)\right| \leq f(n) \mathbb{P}(B) \text { a.s. }
$$

- By setting $1\{B\}=U_{n+m}$, it is easy to see that any $*$-mixing sequence is a $*$-submixing sequence.

Proof of Lemma 12: The proof is based on [29, Theorem 2.20]. There are some important changes to account for the fact that $U_{i n_{0}+j} \notin \sigma\left(\cup_{k=0}^{i}\left(\mathcal{F}_{k n_{0}+j} \backslash \mathcal{F}_{k n_{0}+j-1}\right)\right)$ for some triplet $\left(i, j, n_{0}\right) \in \mathbb{N}^{3}$. The proof in [29, Theorem 2.20] is based on the fact that $U_{i n_{0}+j} \in \sigma\left(\cup_{k=0}^{i}\left(\mathcal{F}_{k n_{0}+j} \backslash \mathcal{F}_{k n_{0}+j-1}\right)\right)$ for any triplet $\left(i, j, n_{0}\right) \in \mathbb{N}^{3}$, which holds when $\mathcal{F}_{n}=\sigma\left(U_{1}^{n}\right)$.

Given $\varepsilon>0$, there exist an $n_{0} \geq N$ such that $f(n)<\varepsilon$ for all $n \geq n_{0}$ since $f(n) \rightarrow 0$ as $n \rightarrow \infty$. From (A.1) we deduce that for all positive integers $i$ and $j$,

$$
\begin{aligned}
\left|\mathbb{E}\left[U_{i n_{0}+j} \mid \mathcal{F}_{(i-1) n_{0}+j}\right]\right| & =\left|\mathbb{E}\left[\mathbb{E}\left[U_{i n_{0}+j} \mid \mathcal{F}_{(i-1) n_{0}+j}\right]-\mathbb{E}\left[U_{i n_{0}+j}\right] \mid \mathcal{F}_{(i-1) n_{0}+j}\right]\right| \\
& \leq \mathbb{E}\left[\left|\mathbb{E}\left[U_{i n_{0}+j} \mid \mathcal{F}_{(i-1) n_{0}+j}\right]-\mathbb{E}\left[U_{i n_{0}+j}\right]\right| \mid \mathcal{F}_{(i-1) n_{0}+j}\right] \\
& \leq \mathbb{E}\left[f\left(n_{0}\right) \mathbb{E}\left|U_{i n_{0}+j}\right| \mid \mathcal{F}_{(i-1) n_{0}+j}\right] \\
& =f\left(n_{0}\right) \mathbb{E}\left|U_{i n_{0}+j}\right|
\end{aligned}
$$

almost surely. Here, (A.6) follows from tower property of conditional expectation [25] and the assumption that $\mathbb{E}\left(U_{n}\right)=0$ for all $n \in \mathbb{N}$, and (A.8) follows from (A.1).

If $n \geq n_{0}$, choose nonnegative integers $q$ and $r$ such that $0 \leq r \leq n_{0}-1$ and $n=q n_{0}+r$. Then,

$$
b_{n}^{-1} \sum_{i=1}^{n} U_{i}=b_{n}^{-1} \sum_{i=1}^{n_{0}} U_{i}+\sum_{j=0}^{n_{0}-1} b_{n}^{-1} \sum_{i=1}^{q-1} U_{i n_{0}+j}+b_{n}^{-1} \sum_{j=0}^{r} U_{q n_{0}+j} .
$$


Observe that

$$
\begin{aligned}
& \sum_{j=0}^{n_{0}-1} b_{n}^{-1} \sum_{i=1}^{q-1} U_{i n_{0}+j}+b_{n}^{-1} \sum_{j=0}^{r} U_{q n_{0}+j} \\
& =\sum_{j=0}^{n_{0}-1} b_{n}^{-1} \sum_{i=1}^{q-1}\left[U_{i n_{0}+j}-\mathbb{E}\left[U_{i n_{0}+j} \mid \mathcal{F}_{(i-1) n_{0}+j}\right]\right] \\
& \quad+\sum_{j=0}^{r} b_{n}^{-1}\left[U_{q n_{0}+j}-\mathbb{E}\left[U_{q n_{0}+j} \mid \mathcal{F}_{(q-1) n_{0}+j}\right]\right] \\
& \quad+b_{n}^{-1} \sum_{j=0}^{n_{0}-1} \sum_{i=1}^{q-1} \mathbb{E}\left[U_{i n_{0}+j} \mid \mathcal{F}_{(i-1) n_{0}+j}\right]+b_{n}^{-1} \sum_{j=0}^{r} \mathbb{E}\left[U_{q n_{0}+j} \mid \mathcal{F}_{(q-1) n_{0}+j}\right] \\
& \leq \sum_{j=0}^{n_{0}-1} \sum_{i=1}^{q-1} b_{n}^{-1}\left[U_{i n_{0}+j}-\mathbb{E}\left[U_{i n_{0}+j} \mid \mathcal{F}_{(i-1) n_{0}+j}\right]\right] \\
& \quad+\sum_{j=0}^{r} b_{n}^{-1}\left[U_{q n_{0}+j}-\mathbb{E}\left[U_{q n_{0}+j} \mid \mathcal{F}_{(q-1) n_{0}+j}\right]\right] \\
& \quad+f\left(n_{0}\right) b_{n}^{-1} \sum_{i=n_{0}}^{n} \mathbb{E}\left|U_{i}\right|
\end{aligned}
$$

where (A.12) follows from (A.9).

Hence, we obtain

$$
\begin{aligned}
b_{n}^{-1} \sum_{i=1}^{n} U_{i} \leq & b_{n}^{-1} \sum_{i=1}^{n_{0}-1} U_{i}+\sum_{j=0}^{n_{0}-1} \sum_{i=1}^{q-1} b_{n}^{-1}\left[U_{i n_{0}+j}-\mathbb{E}\left[U_{i n_{0}+j} \mid \mathcal{F}_{(i-1) n_{0}+j}\right]\right] \\
& +\sum_{j=0}^{r} b_{n}^{-1}\left[U_{q n_{0}+j}-\mathbb{E}\left[U_{q n_{0}+j} \mid \mathcal{F}_{(q-1) n_{0}+j}\right]\right] \\
& +f\left(n_{0}\right) b_{n}^{-1} \sum_{i=n_{0}}^{n} \mathbb{E}\left|U_{i}\right| .
\end{aligned}
$$

Since $n_{0}$ is a fixed number, it is easy to see that the first term on the right-hand side of (A.13) almost surely converges to zero as $n \rightarrow \infty$, i.e.,

$$
\lim _{n \rightarrow \infty} b_{n}^{-1} \sum_{i=1}^{n_{0}} U_{i}=0, \quad \text { a.s. }
$$

Now, for each fixed pair $j \in\left\{0,1,2, \ldots, n_{0}-1\right\}$ and $i \in \mathbb{N}$ denote by

$$
\begin{aligned}
W_{j, i} & =U_{i n_{0}+j}-\mathbb{E}\left[U_{i n_{0}+j} \mid \mathcal{F}_{(i-1) n_{0}+j}\right] \\
V_{j, i} & =b_{i n_{0}+j} .
\end{aligned}
$$

Then, we have $V_{j, i} \in \mathcal{F}_{(i-1) n_{0}+j}$ for all $i, j$. On the other hand, since $U_{n} \in \mathcal{F}_{n}$ for all $n=0,1,2, \ldots$ we also have

$$
W_{j, i}=U_{i n_{0}+j}-\mathbb{E}\left[U_{i n_{0}+j} \mid \mathcal{F}_{(i-1) n_{0}+j}\right] \in \mathcal{F}_{i n_{0}+j}
$$


It follows that for each fixed $j \in\left\{0,1,2, \ldots, n_{0}-1\right\}$ we have

$$
\begin{aligned}
& \mathbb{E}\left[\sum_{i=1}^{q-1} W_{j, i} \mid \mathcal{F}_{(q-2) n_{0}+j}\right] \\
& =\mathbb{E}\left[\sum_{i=1}^{q-2} W_{j, i} \mid \mathcal{F}_{(q-2) n_{0}+j}\right]+\mathbb{E}\left[W_{j, q-1} \mid \mathcal{F}_{(q-2) n_{0}+j}\right] \\
& =\sum_{i=1}^{q-2} W_{j, i}+\mathbb{E}\left[W_{j, q-1} \mid \mathcal{F}_{(q-2) n_{0}+j}\right] \\
& =\sum_{i=1}^{q-2} W_{j, i}+\mathbb{E}\left[U_{(q-1) n_{0}+j}-\mathbb{E}\left[U_{(q-1) n_{0}+j} \mid \mathcal{F}_{(q-2) n_{0}+j}\right] \mid \mathcal{F}_{(q-2) n_{0}+j}\right] \\
& =\sum_{i=1}^{q-2} W_{j, i},
\end{aligned}
$$

or that $\left\{\left(\sum_{i=1}^{q-1} W_{j, i}, \mathcal{F}_{(q-1) n_{0}+j}\right)\right\}_{q=1}^{\infty}$ forms a martingale. In addition, we also have

$$
\begin{aligned}
& \sum_{i=1}^{\infty} V_{j, i}^{-2} \mathbb{E}\left[W_{j, i}^{2} \mid \mathcal{F}_{(i-1) n_{0}+j}\right] \\
& =\sum_{i=1}^{\infty} b_{i n_{0}+j}^{-2} \mathbb{E}\left[\left(U_{i n_{0}+j}-\mathbb{E}\left[U_{i n_{0}+j} \mid \mathcal{F}_{(i-1) n_{0}+j}\right]\right)^{2} \mid \mathcal{F}_{(i-1) n_{0}+j}\right] \\
& \leq \sum_{i=1}^{\infty} b_{i n_{0}+j}^{-2} \mathbb{E}\left[U_{i n_{0}+j}^{2} \mid \mathcal{F}_{(i-1) n_{0}+j}\right]
\end{aligned}
$$

Hence, we obtain that for each fixed $j \in\left\{0,1,2, \ldots, n_{0}-1\right\}$ that

$$
\begin{aligned}
\mathbb{E}\left[\sum_{i=1}^{\infty} V_{j, i}^{-2} \mathbb{E}\left[W_{j, i}^{2} \mid \mathcal{F}_{(i-1) n_{0}+j}\right]\right] & =\mathbb{E}\left[\sum_{i=1}^{\infty} b_{i n_{0}+j}^{-2} \mathbb{E}\left[U_{i n_{0}+j}^{2} \mid \mathcal{F}_{(i-1) n_{0}+j}\right]\right] \\
& =\sum_{i=1}^{\infty} b_{i n_{0}+j}^{-2} \mathbb{E}\left[U_{i n_{0}+j}^{2}\right] \\
& \leq \sum_{n=1}^{\infty} b_{n}^{-2} \mathbb{E}\left[U_{n}^{2}\right] \\
& <\infty .
\end{aligned}
$$

Therefore, it holds almost surely for each fixed $j \in\left\{0,1,2, \ldots, n_{0}-1\right\}$ that

$$
\sum_{i=1}^{\infty} V_{j, i}^{-2} \mathbb{E}\left[W_{j, i}^{2} \mid \mathcal{F}_{(i-1) n_{0}+j}\right]<\infty
$$

This means that the sequences of random variables $\left\{W_{j, i}\right\}_{i=1}^{\infty}$ and $\left\{V_{j, i}\right\}_{i=1}^{\infty}$ satisfy (A.3) in Lemma 11. It follows that as $q \rightarrow \infty$ we have

$$
b_{(q-1) n_{0}+j}^{-1} \sum_{i=1}^{q-1}\left[U_{i n_{0}+j}-\mathbb{E}\left[U_{i n_{0}+j} \mid \mathcal{F}_{(i-1) n_{0}+j}\right]\right]=V_{j, q-1}^{-1} \sum_{i=1}^{q-1} W_{j, i} \rightarrow 0, \quad \text { a.s. }
$$

for each fixed $j \in\left\{0,1,2, \ldots, n_{0}-1\right\}$. It follows that as $q \rightarrow \infty$

$$
b_{(q-1) n_{0}+j}^{-1}\left|\sum_{i=1}^{q-1}\left[U_{i n_{0}+j}-\mathbb{E}\left[U_{i n_{0}+j} \mid \mathcal{F}_{(i-1) n_{0}+j}\right]\right]\right| \rightarrow 0, \quad \text { a.s. }
$$

for each fixed $j \in\left\{0,1,2, \ldots, n_{0}-1\right\}$. Therefore, we obtain

$$
\sum_{j=0}^{n_{0}-1} b_{(q-1) n_{0}+j}^{-1}\left|\sum_{i=1}^{q-1}\left[U_{i n_{0}+j}-\mathbb{E}\left[U_{i n_{0}+j} \mid \mathcal{F}_{(i-1) n_{0}+j}\right]\right]\right| \rightarrow 0, \quad \text { a.s. }
$$


Hence, we have that as $q \rightarrow \infty$

$$
\begin{aligned}
\left|\sum_{j=0}^{n_{0}-1} \sum_{i=1}^{q-1} b_{n}^{-1}\left[U_{i n_{0}+j}-\mathbb{E}\left[U_{i n_{0}+j} \mid \mathcal{F}_{(i-1) n_{0}+j}\right]\right]\right| & \leq \sum_{j=0}^{n_{0}-1} b_{n}^{-1}\left|\sum_{i=1}^{q-1}\left[U_{i n_{0}+j}-\mathbb{E}\left[U_{i n_{0}+j} \mid \mathcal{F}_{(i-1) n_{0}+j}\right]\right]\right| \\
& \leq \sum_{j=0}^{n_{0}-1} b_{(q-1) n_{0}+j}^{-1}\left|\sum_{i=1}^{q-1}\left[U_{i n_{0}+j}-\mathbb{E}\left[U_{i n_{0}+j} \mid \mathcal{F}_{(i-1) n_{0}+j}\right]\right]\right| \\
& \rightarrow 0, \text { a.s. }
\end{aligned}
$$

Here, (A.33) follows from the fact that $\left\{b_{n}\right\}_{n=1}^{\infty}$ is an increasing sequence. This means that

$$
\sum_{j=0}^{n_{0}-1} \sum_{i=1}^{q-1} b_{n}^{-1}\left[U_{i n_{0}+j}-\mathbb{E}\left[U_{i n_{0}+j} \mid \mathcal{F}_{(i-1) n_{0}+j}\right]\right] \rightarrow 0, \quad \text { a.s. }
$$

Similarly, since for each fixed $j \in\{0,1,2, \ldots, r\}$ we have

$$
\begin{aligned}
& \sum_{q=1}^{\infty} b_{q n_{0}+j}^{-2} \mathbb{E}\left[\left(U_{q n_{0}+j}-\mathbb{E}\left[U_{q n_{0}+j} \mid \mathcal{F}_{(q-1) n_{0}+j}\right]\right)^{2}\right] \\
& =\mathbb{E}\left[\sum_{q=1}^{\infty} b_{q n_{0}+j}^{-2} \mathbb{E}\left[\left(U_{q n_{0}+j}-\mathbb{E}\left[U_{q n_{0}+j} \mid \mathcal{F}_{(q-1) n_{0}+j}\right]\right)^{2} \mid \mathcal{F}_{(q-1) n_{0}+j}\right]\right] \\
& \leq \sum_{q=1}^{\infty} b_{q n_{0}+j}^{-2} \mathbb{E}\left[\mathbb{E}\left[U_{q n_{0}+j}^{2} \mid \mathcal{F}_{(q-1) n_{0}+j}\right]\right] \\
& =\sum_{q=1}^{\infty} b_{q n_{0}+j}^{-2} \mathbb{E}\left[U_{q n_{0}+j}^{2}\right] \\
& \leq \sum_{n=1}^{\infty} b_{n}^{-2} \mathbb{E}\left[U_{n}^{2}\right] \\
& <\infty
\end{aligned}
$$

where (A.36) follows from the monotone convergence theorem [25]. Thus, as $q \rightarrow \infty$

$$
b_{q n_{0}+j}^{-1}\left[U_{q n_{0}+j}-\mathbb{E}\left[U_{q n_{0}+j} \mid \mathcal{F}_{(q-1) n_{0}+j}\right]\right] \rightarrow 0, \quad \text { a.s. }
$$

This holds because for a sequence of random variables $\left\{X_{n}\right\}_{n=1}^{\infty}$, if $\sum_{n=1}^{\infty} \mathbb{E}\left[X_{n}^{2}\right]<\infty$, then $X_{n} \rightarrow 0$ almost surely. This follows from a simple application of Chebyshev's inequality and the (first) Borel-Cantelli lemma. Furthermore, since the positive sequence $\left\{b_{n}\right\}_{n=1}^{\infty}$ is increasing,

$$
b_{n}^{-1}\left|U_{q n_{0}+j}-\mathbb{E}\left[U_{q n_{0}+j} \mid \mathcal{F}_{(q-1) n_{0}+j}\right]\right| \rightarrow 0, \quad \text { a.s. }
$$

for each fixed $j \in\{0,1,2, \ldots, r\}$. It follows that

$$
\begin{aligned}
\left|\sum_{j=0}^{r} b_{n}^{-1}\left[U_{q n_{0}+j}-\mathbb{E}\left[U_{q n_{0}+j} \mid \mathcal{F}_{(q-1) n_{0}+j}\right]\right]\right| & \leq \sum_{j=0}^{r} b_{n}^{-1}\left|U_{q n_{0}+j}-\mathbb{E}\left[U_{q n_{0}+j} \mid \mathcal{F}_{(q-1) n_{0}+j}\right]\right| \\
& \leq \sum_{j=0}^{n_{0}-1} b_{n}^{-1}\left|U_{q n_{0}+j}-\mathbb{E}\left[U_{q n_{0}+j} \mid \mathcal{F}_{(q-1) n_{0}+j}\right]\right| \rightarrow 0, \quad \text { a.s. }
\end{aligned}
$$

Hence, we obtain

$$
b_{n}^{-1} \sum_{j=0}^{r}\left[U_{q n_{0}+j}-\mathbb{E}\left[U_{q n_{0}+j} \mid \mathcal{F}_{(q-1) n_{0}+j}\right]\right] \rightarrow 0, \quad \text { a.s. }
$$


In addition, since $\sup _{n} b_{n}^{-1} \sum_{i=1}^{n} \mathbb{E}\left|U_{i}\right|<\infty$, we have

$$
\begin{aligned}
f\left(n_{0}\right) b_{n}^{-1} \sum_{i=n_{0}}^{n} \mathbb{E}\left|U_{i}\right| & <f\left(n_{0}\right) b_{n}^{-1} \sum_{i=1}^{n} \mathbb{E}\left|U_{i}\right| \\
& \leq f\left(n_{0}\right) \sup _{n} b_{n}^{-1} \sum_{i=1}^{n} \mathbb{E}\left|U_{i}\right| \\
& <\varepsilon \sup _{n} b_{n}^{-1} \sum_{i=1}^{n} \mathbb{E}\left|U_{i}\right| .
\end{aligned}
$$

Combining (A.13), (A.14), (A.35), (A.45), and (A.48) we obtain

$$
\limsup _{n \rightarrow \infty}\left|b_{n}^{-1} \sum_{i=1}^{n} U_{i}\right|<\varepsilon\left(\sup _{n} b_{n}^{-1} \sum_{i=1}^{n} \mathbb{E}\left|U_{i}\right|\right), \quad \text { a.s. }
$$

Take $\varepsilon \rightarrow 0$, we have (A.4), which completes the proof of Lemma 12 .

Lemma 13. Let $K$ and $K^{\prime}$ be two positive constants and let $\left\{\xi_{n}\right\}_{n=0}^{\infty}$ be a sequence adapted to filtration $\left\{\mathcal{F}_{n}\right\}_{n=0}^{\infty}$ such that

$$
\begin{aligned}
\mathbb{E}\left(\xi_{n+1} \mid \mathcal{F}_{n}\right) & \geq \xi_{n}+K, \\
\left|\xi_{n+1}-\xi_{n}\right| & \leq K^{\prime} .
\end{aligned}
$$

Let $\tau$ be a stopping time given by (19) for some $T \in \mathbb{R}$. Then, we have

$$
\mathbb{P}(\tau<\infty)=1
$$

Proof of Lemma 13: We have

$$
\begin{aligned}
\xi_{n} & =\xi_{0}+\sum_{i=1}^{n}\left(\xi_{i}-\xi_{i-1}\right) \\
& =\xi_{0}+\sum_{i=1}^{n} \mathbb{E}\left[\xi_{i}-\xi_{i-1} \mid \mathcal{F}_{i-1}\right]+\sum_{i=1}^{n}\left[\xi_{i}-\xi_{i-1}-\mathbb{E}\left[\xi_{i}-\xi_{i-1} \mid \mathcal{F}_{i-1}\right]\right] \\
& \geq \xi_{0}+n K+\sum_{i=1}^{n} \beta_{i},
\end{aligned}
$$

where $\beta_{i}=\xi_{i}-\xi_{i-1}-\mathbb{E}\left[\xi_{i}-\xi_{i-1} \mid \mathcal{F}_{i-1}\right]$ and (A.55) follows from (A.50).

Obviously, we have from (A.51) that

$$
\begin{aligned}
\mathbb{E}\left[\beta_{i} \mid \mathcal{F}_{i-1}\right] & =0, \\
\mathbb{E}\left[\beta_{i}^{2} \mid \mathcal{F}_{i-1}\right] & \leq \mathbb{E}\left[\left(\xi_{i}-\xi_{i-1}\right)^{2} \mid \mathcal{F}_{i-1}\right] \leq\left(K^{\prime}\right)^{2}, \quad \forall i \in \mathbb{N} .
\end{aligned}
$$

It is easy to see that the sequence $\left\{\beta_{n}\right\}_{n=1}^{\infty}$ is $*$-submixing adapted to $\left\{\mathcal{F}_{n}\right\}_{n=1}^{\infty}$. Indeed, for $m, n \in \mathbb{N}$ we have

$$
\begin{aligned}
\mathbb{E}\left[\beta_{n+m} \mid \mathcal{F}_{n}\right] & =\mathbb{E}\left[\mathbb{E}\left[\beta_{n+m} \mid \mathcal{F}_{n+m-1}\right] \mid \mathcal{F}_{m}\right] \\
& =\mathbb{E}\left[0 \mid \mathcal{F}_{m}\right]=0 .
\end{aligned}
$$

Here, (A.59) follows from (A.56). It follows from (A.59) that $\mathbb{E}\left[\beta_{n+m}\right]=0$. Applying Lemma 12 for the $*$-submixing sequence $\left\{\beta_{n}\right\}_{n=1}^{\infty}$ adapted to $\left\{\mathcal{F}_{n}\right\}_{n=1}^{\infty}$ and $b_{n}=n$ for all $n \in \mathbb{N}$, we obtain

$$
\lim _{n \rightarrow \infty} \frac{1}{n} \sum_{i=1}^{n} \beta_{i}=0, \quad \text { a.s. }
$$

It follows that

$$
\liminf _{n \rightarrow \infty} \frac{\xi_{n}}{n} \geq K>0, \quad \text { a.s. }
$$

Hence, $\mathbb{P}(\tau<\infty)=1$. 


\section{APPENDIX B \\ PROOF OF LEMMA 1}

Before proving Lemma 1, we state some properties of $\xi_{\tau}$ and $\xi_{n \wedge \tau}$ in the following lemma.

Lemma 14. Under the same assumptions as Lemma 13, we have

- $\xi_{\tau}$, which is defined in Lemma 1, is a random variable, i.e., measurable with respect to $\mathcal{F}_{\infty}=\cup_{n=1}^{\infty} \mathcal{F}_{n}=\lim _{n \rightarrow \infty} \mathcal{F}_{n}$.

- The following limiting statement holds:

$$
\lim _{n \rightarrow \infty} \mathbb{E}\left(\left|\xi_{n \wedge \tau}\right|\right)=\mathbb{E}\left(\left|\xi_{\tau}\right|\right)
$$

Proof of Lemma 14: Applying Lemma 13 with the identifications $K=\min \left\{K_{1}, K_{2}\right\}$ and $K^{\prime}=K_{3}\left(K_{1}, K_{2}\right.$, and $K_{3}$ are defined in Lemma 1), we have $\mathbb{P}(\tau<\infty)=1$, hence $\xi_{n \wedge \tau}$ almost surely converges to $\xi_{\tau}$ as $n \rightarrow \infty$. This means that $\xi_{\tau}$ is a well-defined random variable since the limit of a sequence of Borel measurable functions is a Borel measurable function [30]. In addition, from Lemma 13 we also have $\mathbb{E}(\tau)<\infty$. Now, observe that

$$
\xi_{n \wedge \tau}=\xi_{0}+\sum_{i=0}^{n \wedge \tau-1}\left(\xi_{i+1}-\xi_{i}\right) .
$$

Define

$$
\xi_{\max }=\left|\xi_{0}\right|+\sum_{i=0}^{\infty}\left|\xi_{i+1}-\xi_{i}\right| \mathbf{1}\{\tau>i\} .
$$

It is easy to see that $\left|\xi_{n \wedge \tau}\right| \leq \xi_{\max }$ for all $n$. Now, we also have

$$
\begin{aligned}
\mathbb{E}\left[\xi_{\text {max }}\right] & =\mathbb{E}\left[\left|\xi_{0}\right|+\sum_{i=0}^{\infty}\left|\xi_{i+1}-\xi_{i}\right| \mathbf{1}\{\tau>i\}\right] \\
& =\mathbb{E}\left[\left|\xi_{0}\right|\right]+\mathbb{E}\left[\sum_{i=0}^{\infty}\left|\xi_{i+1}-\xi_{i}\right| \mathbf{1}\{\tau>i\}\right] \\
& \leq \mathbb{E}\left[\left|\xi_{0}\right|\right]+K_{3} \sum_{i=0}^{\infty} \mathbb{E}[\mathbf{1}\{\tau>i\}] \\
& =\mathbb{E}\left[\left|\xi_{0}\right|\right]+K_{3} \sum_{i=0}^{\infty} \mathbb{P}(\tau>i) \\
& =\mathbb{E}\left[\left|\xi_{0}\right|\right]+K_{3} \mathbb{E}(\tau) \\
& <\infty
\end{aligned}
$$

where (B.6) follows from (18) and Fatou's lemma [25]. Since $\left|\xi_{n \wedge \tau}\right| \rightarrow\left|\xi_{\tau}\right|$ as $n \rightarrow \infty$, (B.1) is obtained by the dominated convergence theorem [25].

We are now ready to prove Lemma 1.

Proof of Lemma 1: The proof idea is based on [26]. However, the submartingale construction is changed to account for all the points in Remark 2. First, we choose $G \in \mathbb{R}$ such that the following equality holds:

$$
G=\left|\frac{1}{K_{2}}-\frac{1}{K_{1}}\right| \cdot \max _{-K_{3} \leq x \leq K_{3}}\left[\frac{x}{\exp (x)-1}\right] .
$$

It is easy to see that $G>0$. Now, define the following sequence

$$
\eta_{n}= \begin{cases}-G+G \exp \left(\xi_{n}\right)+\frac{\xi_{n}}{K_{1}}-n, & \text { if } \quad \xi_{n}<0 \\ \frac{\xi_{n}}{K_{2}}-n+\frac{K_{2}}{K_{1}}-1, & \text { if } \quad \xi_{n} \geq 0\end{cases}
$$

where $G$ are defined in (B.10). First, we show that $\left(\eta_{n}, \mathcal{F}_{n}\right)$ forms a submartingale, i.e.

$$
\mathbb{E}\left(\eta_{n+1} \mid \mathcal{F}_{n}\right) \geq \eta_{n}, \quad n=0,1,2, \ldots
$$

We consider four different cases. 
- Case 1: For $\xi_{n} \geq 0$ and $\xi_{n+1} \geq 0$, we obtain

$$
\begin{aligned}
\mathbb{E}\left[\eta_{n+1} \mid \mathcal{F}_{n}\right] & =\mathbb{E}\left[\frac{\xi_{n+1}}{K_{2}}-(n+1)+\frac{K_{2}}{K_{1}}-1 \mid \mathcal{F}_{n}\right] \\
& \geq \frac{\left(\xi_{n}+K_{2}\right)}{K_{2}}-(n+1)+\frac{K_{2}}{K_{1}}-1 \\
& =\frac{\xi_{n}}{K_{2}}-n+\frac{K_{2}}{K_{1}}-1 \\
& =\eta_{n} .
\end{aligned}
$$

Here, (B.14) follows from (17) and (B.16) follows from (B.11).

- Case 2: For $\xi_{n} \geq 0$ and $\xi_{n+1}<0$, we obtain

$$
\begin{aligned}
\mathbb{E}\left[\eta_{n+1} \mid \mathcal{F}_{n}\right] & =\mathbb{E}\left[-G+G \exp \left(\xi_{n+1}\right)+\frac{\xi_{n+1}}{K_{1}}-(n+1) \mid \mathcal{F}_{n}\right] \\
& \geq-G+G \exp \left(\mathbb{E}\left[\xi_{n+1} \mid \mathcal{F}_{n}\right]\right)+\frac{1}{K_{1}} \mathbb{E}\left[\xi_{n+1} \mid \mathcal{F}_{n}\right]-(n+1) \\
& \geq-G+G \exp \left(\xi_{n}+K_{2}\right)+\frac{1}{K_{1}}\left(\xi_{n}+K_{2}\right)-(n+1) \\
& \geq-G+G \exp \left(\xi_{n}\right)+\frac{1}{K_{1}} \xi_{n}-n+\frac{K_{2}}{K_{1}}-1 \\
& \geq \frac{\xi_{n}}{K_{2}}-n+\frac{K_{2}}{K_{1}}-1 \\
& =\eta_{n}
\end{aligned}
$$

Here, (B.18) follows from the convexity of $\exp (x)$, (B.19) follows from (17), (B.21) follows from the fact $0 \leq \xi_{n} \leq$ $\xi_{n+1}+K_{3}<K_{3}$ and (B.10), and (B.22) follows from (B.11).

- Case 3: For $\xi_{n}<0, \xi_{n+1} \geq 0$, we have

$$
\begin{aligned}
\mathbb{E}\left[\eta_{n+1} \mid \mathcal{F}_{n}\right] & =\mathbb{E}\left[\frac{\xi_{n+1}}{K_{2}}-(n+1)+\frac{K_{2}}{K_{1}}-1 \mid \mathcal{F}_{n}\right] \\
& \geq \frac{\left(\xi_{n}+K_{1}\right)}{K_{2}}-(n+1)+\frac{K_{2}}{K_{1}}-1 \\
& =\frac{\xi_{n}}{K_{2}}-n+\frac{K_{1}}{K_{2}}+\frac{K_{2}}{K_{1}}-2 \\
& \geq \frac{\xi_{n}}{K_{2}}-n \\
& \geq-G+G \exp \left(\xi_{n}\right)+\frac{\xi_{n}}{K_{1}}-n \\
& =\eta_{n} .
\end{aligned}
$$

Here, (B.24) follows from (16), (B.26) follows from the fact that $\frac{K_{1}}{K_{2}}+\frac{K_{2}}{K_{1}} \geq 2$, for any $K_{1}, K_{2}>0$, (B.27) follows from the fact that $0>\xi_{n} \geq \xi_{n+1}-K_{3} \geq-K_{3}$ and (B.10), and (B.28) follows from (B.11).

- Case 4: For the case $\xi_{n}<0, \xi_{n+1}<0$, we have

$$
\begin{aligned}
\mathbb{E} & {\left[-G+G \exp \left(\xi_{n+1}\right)+\frac{\xi_{n+1}}{K_{1}}-(n+1) \mid \mathcal{F}_{n}\right] } \\
= & \mathbb{E}\left[\frac{\xi_{n+1}}{K_{1}}-\frac{\xi_{n}}{K_{1}}+G \exp \left(\xi_{n+1}\right)-G \exp \left(\xi_{n}\right) \mid \mathcal{F}_{n}\right] \\
& -G+G \exp \left(\xi_{n}\right)+\frac{\xi_{n}}{K_{1}}-(n+1) \\
= & \eta_{n}-1+\frac{1}{K_{1}} \mathbb{E}\left[\xi_{n+1}-\xi_{n} \mid \mathcal{F}_{n}\right]+G \exp \left(\xi_{n}\right) \mathbb{E}\left[\exp \left(\xi_{n+1}-\xi_{n}\right)-1 \mid \mathcal{F}_{n}\right] \\
\geq & \eta_{n}-1+\frac{1}{K_{1}} \mathbb{E}\left[\xi_{n+1}-\xi_{n} \mid \mathcal{F}_{n}\right]+G \exp \left(\xi_{n}\right) \mathbb{E}\left[\xi_{n+1}-\xi_{n} \mid \mathcal{F}_{n}\right] \\
\geq & \eta_{n}+G \exp \left(\xi_{n}\right) K_{1} \\
\geq & \eta_{n} .
\end{aligned}
$$

Here, (B.31) follows from (B.11), (B.32) follows from the fact that $\exp (x)-1 \geq x$, and (B.33) follows from (16). 
Now, from (B.11) we have

$$
\begin{aligned}
\eta_{n \wedge \tau}= & {\left[\frac{\xi_{n \wedge \tau}}{K_{2}}-n \wedge \tau+\frac{K_{2}}{K_{1}}-1\right] \mathbf{1}\left\{\xi_{n \wedge \tau} \geq 0\right\} } \\
& +\left[-G+G \exp \left(\xi_{n \wedge \tau}\right)+\frac{\xi_{n \wedge \tau}}{K_{1}}-n \wedge \tau\right] \mathbf{1}\left\{\xi_{n \wedge \tau}<0\right\} \\
= & \left.\frac{K_{2}}{K_{1}}-1+\frac{\xi_{n \wedge \tau}}{K_{2}}\right] \mathbf{1}\left\{\xi_{n \wedge \tau} \geq 0\right\} \\
& +\left[-G+G \exp \left(\xi_{n \wedge \tau}\right)+\frac{\xi_{n \wedge \tau}}{K_{1}}\right] \mathbf{1}\left\{\xi_{n \wedge \tau}<0\right\}-(n \wedge \tau) \\
\leq & \frac{\xi_{n \wedge \tau}}{K_{2}} \mathbf{1}\left\{\xi_{n \wedge \tau} \geq 0\right\}+\frac{\xi_{n \wedge \tau}}{K_{1}} \mathbf{1}\left\{\xi_{n \wedge \tau}<0\right\}-(n \wedge \tau) \\
& +\left(\frac{K_{2}}{K_{1}}-1\right) \mathbf{1}\left\{\xi_{n \wedge \tau} \geq 0\right\}+\left(-G+G \exp \left(\xi_{n \wedge \tau}\right)\right) \mathbf{1}\left\{\xi_{n \wedge \tau}<0\right\} \\
\leq & \frac{\xi_{n \wedge \tau}}{K_{2}} \mathbf{1}\left\{\xi_{n \wedge \tau} \geq 0\right\}+\frac{\xi_{n \wedge \tau}}{K_{1}} \mathbf{1}\left\{\xi_{n \wedge \tau}<0\right\}-(n \wedge \tau) \\
& +\left(\frac{K_{2}}{K_{1}}-1\right) \mathbf{1}\left\{\xi_{n \wedge \tau} \geq 0\right\} \\
\leq & \frac{\xi_{n \wedge \tau}}{K_{2}} \mathbf{1}\left\{\xi_{n \wedge \tau} \geq 0\right\}+\frac{\xi_{n \wedge \tau}}{K_{1}} \mathbf{1}\left\{\xi_{n \wedge \tau}<0\right\}-(n \wedge \tau)+\left|\frac{K_{2}}{K_{1}}-1\right| \\
\leq & \frac{\xi_{n \wedge \tau}}{K_{2}} \mathbf{1}\left\{\xi_{n \wedge \tau} \geq 0\right\}-(n \wedge \tau)+\left|\frac{K_{2}}{K_{1}}-1\right| \\
\leq & \frac{\left|\xi_{n \wedge \tau}\right|}{K_{2}}-(n \wedge \tau)+\left|\frac{K_{2}}{K_{1}}-1\right| .
\end{aligned}
$$

Here, (B.38) follows from the fact that $(-G+G \exp (x)) \mathbf{1}\{x<0\} \leq 0$. Hence, from (B.12) we obtain

$$
\begin{aligned}
\eta_{0} & \leq \mathbb{E}\left(\eta_{n \wedge \tau}\right) \\
& \leq \limsup _{n \rightarrow \infty} \mathbb{E}\left(\eta_{n \wedge \tau}\right) \\
& \leq \limsup _{n \rightarrow \infty}\left[\frac{\left|\xi_{n \wedge \tau}\right|}{K_{2}}-(n \wedge \tau)+\left|\frac{K_{2}}{K_{1}}-1\right|\right] \\
& \leq \frac{\mathbb{E}\left[\left|\xi_{\tau}\right|\right]}{K_{2}}-\mathbb{E}(\tau)+\left|\frac{K_{2}}{K_{1}}-1\right| \\
& \leq \frac{|T|+K_{3}}{K_{2}}-\mathbb{E}(\tau)+\left|\frac{K_{2}}{K_{1}}-1\right| .
\end{aligned}
$$

Here, (B.45) follows from Lemma 14 and (B.46) follows from from the fact that $T \leq \xi_{\tau} \leq T+K_{3}$, so $\left|\xi_{\tau}\right| \leq \max \{|T|, \mid T+$ $\left.K_{3} \mid\right\} \leq|T|+K_{3}$. This means that

$$
\begin{aligned}
\mathbb{E}(\tau) & \leq \frac{|T|+K_{3}}{K_{2}}-\eta_{0}+\left|\frac{K_{2}}{K_{1}}-1\right| \\
& =\frac{|T|+K_{3}}{K_{2}}-\left(-G+G \exp \left(\xi_{0}\right)+\frac{\xi_{0}}{K_{1}}\right) \mathbf{1}\left\{\xi_{0}<0\right\}-\left(\frac{\xi_{0}}{K_{2}}+\frac{K_{2}}{K_{1}}-1\right) \mathbf{1}\left\{\xi_{0} \geq 0\right\}+\left|\frac{K_{2}}{K_{1}}-1\right| \\
& \leq \frac{|T|+K_{3}}{K_{2}}+G-\frac{\xi_{0}}{K_{1}} \mathbf{1}\left\{\xi_{0}<0\right\}-\frac{\xi_{0}}{K_{2}} \mathbf{1}\left\{\xi_{0} \geq 0\right\}+2\left|\frac{K_{2}}{K_{1}}-1\right| \\
& =-K_{1}^{-1} \xi_{0} \mathbf{1}\left\{\xi_{0}<0\right\}-K_{2}^{-1} \xi_{0} \mathbf{1}\left\{\xi_{0} \geq 0\right\}+K_{2}^{-1}|T|+f\left(K_{1}, K_{2}, K_{3}\right) .
\end{aligned}
$$

Here, (B.48) follows from (B.11) and $f$ is some function of $K_{1}, K_{2}$, and $K_{3}$. This concludes the proof of Lemma 1 .

APPENDIX C

PROOF OF LEMMA 2

Define the sequence of random variables

$$
\gamma_{n}=\xi_{n}+K_{2} n, \quad n=0,1,2, \ldots
$$


It follows from (22) that

$$
\begin{aligned}
\gamma_{n} & \in \mathcal{F}_{n}, \quad n=0,1,2, \ldots, \\
\mathbb{E}\left(\gamma_{n+1} \mid \mathcal{F}_{n}\right) & \leq \gamma_{n}, \quad \forall n \geq \tau_{0} .
\end{aligned}
$$

Using Lemma 13 with $K=K_{1}$, we know that

$$
\mathbb{P}\left(\tau_{0}<\infty\right)=1
$$

Similarly, from (C.4) and Lemma 13, we also obtain (A.52), i.e.,

$$
\mathbb{P}(\tau<\infty)=1
$$

since the new initial value of the supermartingale starts at $\tau_{0}$, i.e.,

$$
\left|\xi_{\tau_{0}}\right|<\left|T_{0}\right|+K_{3}<\infty
$$

It follows from (C.4) and (C.5) that

$$
\begin{aligned}
\gamma_{\tau_{0}} & =\lim _{n \rightarrow \infty} \gamma_{\tau_{0} \wedge n} \in \mathcal{F}_{\infty}, \\
\gamma_{\tau} & =\lim _{n \rightarrow \infty} \gamma_{\tau \wedge n} \in \mathcal{F}_{\infty} .
\end{aligned}
$$

Now, since $\tau \geq \tau_{0}$, we have for any $n \geq 0$ that

$$
\gamma_{\tau \wedge n}=\gamma_{\tau_{0} \wedge n}+\sum_{k=0}^{n}\left(\gamma_{k+1}-\gamma_{k}\right) \mathbf{1}\left\{\tau_{0} \wedge n \leq k<\tau \wedge n\right\}
$$

Since $\tau_{0}$ is a stopping time of the filtration $\left\{\mathcal{F}_{n}\right\}_{n=0}^{\infty}, \tau_{0} \wedge n$ is also a stopping time of this filtration [25]. Define

$$
\mathcal{F}_{\tau_{0} \wedge n}=\left\{A \in \mathcal{F}_{\infty}: A \cap\left\{\tau_{0} \wedge n \leq k\right\} \in \mathcal{F}_{k}, k=0,1,2, \ldots\right\} .
$$

Now, for any $A \in \mathcal{F}_{\tau_{0} \wedge n}$, it is easy to see that

$$
\begin{aligned}
\mathbf{1}\left\{\tau_{0} \wedge n \leq k<\tau \wedge n\right\} \mathbf{1}\{A\} & =\mathbf{1}\left\{\left\{\tau_{0} \wedge n \leq k\right\} \cap A\right\}-\mathbf{1}\{\{\tau \wedge n \leq k\} \cap A\} \\
& =\mathbf{1}\left\{\left\{\tau_{0} \wedge n \leq k\right\} \cap A\right\}-\mathbf{1}\left\{\{\tau \wedge n \leq k\} \cap\left(\left\{\tau_{0} \wedge n \leq k\right\} \cap A\right)\right\} \\
& \in \mathcal{F}_{k} .
\end{aligned}
$$

Here, (C.12) follows from the assumption that $\tau \geq \tau_{0}$ and (C.13) follows from the fact that $\left\{\tau_{0} \wedge n \leq k\right\} \cap A \in \mathcal{F}_{k}$ for any $A \in \mathcal{F}_{\tau_{0} \wedge n}$ and that $\tau \wedge n$ is a bounded stopping time of the same filtration $\left\{\mathcal{F}_{n}\right\}_{n=0}^{\infty}$, i.e., $\{\tau \wedge n \leq k\} \in \mathcal{F}_{k}[25]$.

It follows from (C.3) that

$$
\mathbb{E}\left[\left(\gamma_{k+1}-\gamma_{k}\right) \mathbf{1}\left\{\tau_{0} \leq k<\tau \wedge n\right\} \mathbf{1}\{A\}\right] \leq 0, \quad \forall k \geq \tau_{0}
$$

Therefore, we have

$$
\begin{aligned}
& \mathbb{E}\left[\sum_{k=0}^{n}\left(\gamma_{k+1}-\gamma_{k}\right) \mathbf{1}\left\{\tau_{0} \wedge n \leq k<\tau \wedge n\right\} \mathbf{1}\{A\}\right] \\
& \quad=\mathbb{E}\left[\sum_{k=0}^{n}\left(\gamma_{k+1}-\gamma_{k}\right) \mathbf{1}\left\{\tau_{0} \wedge n \leq k<\tau_{0}\right\} \mathbf{1}\{A\}\right]+\mathbb{E}\left[\sum_{k=0}^{n}\left(\gamma_{k+1}-\gamma_{k}\right) \mathbf{1}\left\{\tau_{0} \leq k<\tau \wedge n\right\} \mathbf{1}\{A\}\right] \\
& \quad \leq \mathbb{E}\left[\sum_{k=0}^{n}\left(\gamma_{k+1}-\gamma_{k}\right) \mathbf{1}\left\{\tau_{0} \wedge n \leq k<\tau_{0}\right\} \mathbf{1}\{A\}\right]
\end{aligned}
$$

where (C.17) follows from (C.14). Now, observe that

$$
\sum_{k=0}^{n}\left(\gamma_{k+1}-\gamma_{k}\right) \mathbf{1}\left\{\tau_{0} \wedge n \leq k<\tau_{0}\right\} \mathbf{1}\{A\}=\left\{\begin{array}{ll}
0, & \text { if } \tau_{0} \leq n \\
\left(\gamma_{n+1}-\gamma_{n}\right) \mathbf{1}\{A\}, & \text { if } \quad \tau_{0}>n
\end{array} .\right.
$$

Hence, for all $n \geq 0$ we have from (18) and (C.18) that

$$
\sum_{k=0}^{n}\left(\gamma_{k+1}-\gamma_{k}\right) \mathbf{1}\left\{\tau_{0} \wedge n \leq k<\tau_{0}\right\} \mathbf{1}\{A\} \leq K_{3}
$$

From (C.9), (C.17), and (C.19) we have

$$
\mathbb{E}\left(\gamma_{\tau \wedge n} \mathbf{1}\{A\}\right) \leq \mathbb{E}\left(\gamma_{\tau_{0} \wedge n} \mathbf{1}\{A\}\right)+K_{3}
$$


for any $A \in \mathcal{F}_{\tau_{0} \wedge n}$. Since $\mathcal{F}_{\tau_{0} \wedge n}$ is a $\sigma$-algebra [25], therefore, by taking $A$ to be the entire sample space in (C.20), we obtain for any $n \geq 0$ that

$$
\mathbb{E}\left(\gamma_{\tau \wedge n}\right) \leq \mathbb{E}\left(\gamma_{\tau_{0}}\right)+K_{3}
$$

Now, we have for all $n \geq 0$ that

$$
\begin{aligned}
\gamma_{\tau \wedge n} & =\gamma_{0}+\sum_{k=0}^{\tau \wedge n}\left(\gamma_{k+1}-\gamma_{k}\right) \\
& \leq\left|\gamma_{0}\right|+\sum_{k=0}^{\tau \wedge n}\left|\gamma_{k+1}-\gamma_{k}\right| \\
& \leq\left|\gamma_{0}\right|+\sum_{k=0}^{\tau}\left|\gamma_{k+1}-\gamma_{k}\right| \\
& =\left|\gamma_{0}\right|+\sum_{k=0}^{\infty}\left|\gamma_{k+1}-\gamma_{k}\right| \mathbf{1}\{\tau>k\} \\
& =\left|\xi_{0}\right|+\sum_{k=0}^{\infty}\left|\xi_{k+1}-\xi_{k}+K_{2}\right| \mathbf{1}\{\tau>k\} \\
& \leq\left|\xi_{0}\right|+\sum_{k=0}^{\infty}\left(\left|\xi_{k+1}-\xi_{k}\right|+K_{2}\right) \mathbf{1}\{\tau>k\} \\
& \leq\left|\xi_{0}\right|+\left(K_{3}+K_{2}\right) \sum_{k=0}^{\infty} \mathbf{1}\{\tau>k\}
\end{aligned}
$$

Here, (C.26) follows from (C.1), and (C.28) follows from (18). Hence, we have for all $n \geq 0$ that

$$
\begin{aligned}
\mathbb{E}\left|\gamma_{\tau \wedge n}\right| & \leq \mathbb{E}\left|\xi_{0}\right|+\left(K_{2}+K_{3}\right) \sum_{k=0}^{\infty} \mathbb{P}(\tau>k) \\
& =\mathbb{E}\left|\xi_{0}\right|+\left(K_{2}+K_{3}\right) \mathbb{E}(\tau) \\
& <\infty
\end{aligned}
$$

Here, (C.31) follows from (C.5). In addition, from (C.4) and (C.5) we also have

$$
\lim _{n \rightarrow \infty} \gamma_{\tau \wedge n}=\gamma_{\tau}
$$

By the dominated convergence theorem [25] and from (C.8), (C.28), (C.31), and (C.32) we have

$$
\lim _{n \rightarrow \infty} \mathbb{E}\left(\gamma_{\tau \wedge n}\right)=\mathbb{E}\left(\gamma_{\tau}\right)
$$

From (C.21) and (C.33) we obtain

$$
\mathbb{E}\left(\gamma_{\tau}\right) \leq \mathbb{E}\left(\gamma_{\tau_{0}}\right)+K_{3}
$$

Combining (C.1) and (C.34) we have

$$
\mathbb{E}\left(\xi_{\tau}+\tau K_{2}\right) \leq \mathbb{E}\left(\xi_{\tau_{0}}+\tau_{0} K_{2}\right)+K_{3}
$$

Hence, we obtain

$$
\begin{aligned}
\mathbb{E}\left(\tau-\tau_{0}\right) & \leq \frac{\mathbb{E}\left(\xi_{\tau_{0}}-\xi_{\tau}\right)+K_{3}}{K_{2}} \\
& \leq \frac{T_{0}-T+3 K_{3}}{K_{2}} .
\end{aligned}
$$

Here, (C.37) follows from the fact that $\xi_{\tau_{0}} \leq T_{0}+K_{3}$ and that $\xi_{\tau} \geq T-K_{3}$ if $T \leq T_{0}$. This proves (25) since $T_{0} \geq T$. 
APPENDIX D

PROOF OF LEMMA 4

From (31) we have

$$
\begin{aligned}
\liminf _{L \rightarrow \infty} \frac{-\ln \varepsilon_{L}}{L \rho_{L}^{\prime}} & \geq \liminf _{L \rightarrow \infty} \frac{-B}{p_{0, L} L \rho_{L}^{\prime}}\left[-\frac{L \rho_{L}^{\prime}}{C}+\left[\frac{1}{C}-\frac{p_{0, L}}{B}+\frac{3\left(1-p_{0, L}\right)}{2 B^{*}}\right] Z_{0, L}+q_{1}\left(P_{Y \mid X}\right)\right] \\
& =\liminf _{L \rightarrow \infty} \frac{B}{C p_{0, L}}-\frac{B}{p_{0, L}}\left[\frac{1}{C}-\frac{p_{0, L}}{B}+\frac{3\left(1-p_{0, L}\right)}{2 B^{*}}\right] \frac{Z_{0, L}}{L \rho_{L}^{\prime}}-\frac{B q_{1}\left(P_{Y \mid X}\right)}{p_{0, L} L \rho_{L}^{\prime}} .
\end{aligned}
$$

Note that

$$
\begin{aligned}
\lim _{L \rightarrow \infty} p_{0, L} & =\lim _{L \rightarrow \infty} 1-\frac{1}{L}=1 \\
\lim _{L \rightarrow \infty} \frac{Z_{0, L}}{L \rho_{L}^{\prime}} & =\lim _{L \rightarrow \infty} \frac{1}{L \rho_{L}^{\prime}} \ln \left(\frac{p_{0, L}}{1-p_{0, L}}\right)=\lim _{L \rightarrow \infty} \frac{\ln (L-1)}{L \rho_{L}^{\prime}}=0 \\
\lim _{L \rightarrow \infty} \frac{1}{p_{0, L} L \rho_{L}^{\prime}} & =\lim _{L \rightarrow \infty} \frac{1}{\sqrt{L} p_{0, L}\left(\rho_{L}^{\prime} \sqrt{L}\right)}=0 .
\end{aligned}
$$

It follows that

$$
\liminf _{L \rightarrow \infty} \frac{-\ln \varepsilon_{L}}{L \rho_{L}^{\prime}} \geq \frac{B}{C} .
$$

From (D.6) we have

$$
\frac{-\ln \varepsilon_{L}}{L \rho_{L}^{\prime}} \geq \frac{B}{2 C}
$$

for $L$ sufficiently large. This is equivalent to

$$
\varepsilon_{L} \leq \exp \left(-\frac{B}{2 C} L \rho_{L}^{\prime}\right)
$$

for $L$ sufficiently large. Recall the definition of $Z_{0, L}$ in (30). We have for $L$ sufficiently large that

$$
\begin{aligned}
\exp \left(-A_{L}\right)-\varepsilon_{L} \exp \left(-C_{2}\right) & =\exp \left(-Z_{0, L} / 2\right)-\varepsilon_{L} \exp \left(-C_{2}\right) \\
& =\frac{1}{\sqrt{L-1}}-\varepsilon_{L} \exp \left(-C_{2}\right) \\
& \geq \frac{1}{\sqrt{L-1}}-\exp \left(-\frac{B}{2 C} L \rho_{L}^{\prime}\right) \exp \left(-C_{2}\right) \\
& \geq \frac{1}{\sqrt{L-1}}-\exp \left(-\frac{B}{2 C} \sqrt{L}\right) \exp \left(-C_{2}\right) \\
& \geq \frac{1}{2 \sqrt{L-1}} .
\end{aligned}
$$

Here, (D.12) follows from the fact that $\sqrt{L} \rho_{L}^{\prime} \rightarrow \infty$ as $L \rightarrow \infty$. Therefore, we obtain for $L$ sufficiently large that

$$
\begin{aligned}
p_{1, L} & \leq \frac{\exp \left(-Z_{0, L}\right)-\varepsilon_{L} \exp \left(-C_{2}\right)}{\exp \left(-A_{L}\right)-\varepsilon_{L} \exp \left(-C_{2}\right)} \\
& =2 \sqrt{L-1}\left[\exp \left(-Z_{0, L}\right)-\varepsilon_{L} \exp \left(-C_{2}\right)\right] \\
& \leq 2 \sqrt{L-1}\left[\frac{1}{L-1}-\varepsilon_{L} \exp \left(-C_{2}\right)\right] \\
& \leq \frac{2}{\sqrt{L-1}}
\end{aligned}
$$


Hence, we obtain from (34) that

$$
\begin{aligned}
p_{0, L}\left(1-p_{1, L}\right) \mathbb{E}\left(W_{L}\right)= & -p_{0, L} \frac{\ln \varepsilon_{L}}{B}+\frac{\ln \left(\exp \left(L\left(C-\rho_{L}^{\prime}\right)\right)-1\right)}{C} \\
& +\left[\frac{1}{C}-\frac{p_{0, L}}{B}+\frac{\left(1-p_{0, L}\right)}{B^{*}}\right] Z_{0, L}+\frac{\left(1-p_{0, L}\right)\left|A_{L}\right|}{B^{*}}+q_{1}\left(P_{Y \mid X}\right) \\
\leq & -p_{0, L} \frac{\ln \varepsilon_{L}}{B}+\frac{L C-L \rho_{L}^{\prime}}{C}+\left[\frac{1}{C}-\frac{p_{0, L}}{B}+\frac{\left(1-p_{0, L}\right)}{B^{*}}\right] Z_{0, L} \\
& +\frac{\left(1-p_{0, L}\right)\left|A_{L}\right|}{B^{*}}+q_{1}\left(P_{Y \mid X}\right) \\
\leq & L-p_{0, L} \frac{\ln \varepsilon_{L}}{B}-\frac{L \rho_{L}^{\prime}}{C}+\left[\frac{1}{C}-\frac{p_{0, L}}{B}+\frac{3\left(1-p_{0, L}\right)}{2 B^{*}}\right] Z_{0, L}+q_{1}\left(P_{Y \mid X}\right) \\
= & L .
\end{aligned}
$$

Here, (D.20) follows from (32) and (D.21) follows from (31). Hence, from (D.17),

$$
\mathbb{E}\left(W_{L}\right) \leq \frac{L}{p_{0, L}\left(1-p_{1, L}\right)} \leq \frac{L}{(1-1 / L)(1-2 /(\sqrt{L-1}))} \leq L+3 \sqrt{L}
$$

for $L$ sufficiently large.

\section{APPENDIX E}

PROOF OF LEMMA 7

Proof: The proof is based on a combination of Burnashev's arguments in both [4] and [9]. We can assume that $P_{Y \mid X}(y \mid x)>$ 0 for all $x \in \mathcal{X}, y \in \mathcal{Y}$, otherwise (110) trivially holds since $B=\infty$. For each $i=1,2, \ldots, M$ and $y \in \mathcal{Y}$, define

$$
\begin{aligned}
p_{i} & =\mathbb{P}\left(W=i \mid Y^{n}\right), \\
p_{i}(y) & =\mathbb{P}\left(W=i \mid Y^{n}, Y_{n+1}=y\right), \\
p(y \mid W=i) & =\mathbb{P}\left(Y_{n+1}=y \mid Y^{n}, W=i\right), \\
p(y \mid W \neq i) & =\mathbb{P}\left(Y_{n+1}=y \mid Y^{n}, W \neq i\right), \\
p(y) & =\mathbb{P}\left(Y_{n+1}=y \mid Y^{n}\right) .
\end{aligned}
$$

We may assume without loss of generality that $p_{i} \neq 1$ for all $i \in \mathcal{W}=\{1, \ldots, M\}$. Otherwise, again the inequalities in (110) trivially hold. Using [4, Lemma 7] and the definitions in (E.1)-(E.5) we have

$$
\begin{aligned}
\mathbb{E}\left[\ln \mathcal{H}\left(W \mid Y^{n}\right)-\ln \mathcal{H}\left(W \mid Y^{n+1}\right) \mid Y^{n}\right] & =\sum_{y \in \mathcal{Y}} p(y) \ln \left[\frac{-\sum_{i=1}^{M} p_{i} \ln p_{i}}{-\sum_{i=1}^{M} p_{i}(y) \ln p_{i}(y)}\right] \\
& \leq \max _{i}\left\{\sum_{y \in \mathcal{Y}} p(y) \ln \left[\frac{-p_{i} \ln p_{i}}{-p_{i}(y) \ln p_{i}(y)}\right]\right\}
\end{aligned}
$$

Define

$$
F_{i}=\sum_{y \in \mathcal{Y}} p(y) \ln \left[\frac{-p_{i} \ln p_{i}}{-p_{i}(y) \ln p_{i}(y)}\right]
$$

It is easy to see that

$$
\begin{aligned}
p(y) & =p_{i} p(y \mid W=i)+\left(1-p_{i}\right) p(y \mid W \neq i), \\
p_{i}(y) & =\frac{p_{i} p(y \mid W=i)}{p(y)}
\end{aligned}
$$

and

$$
\begin{aligned}
p(y \mid W=i) & =\mathbb{P}\left(Y_{n+1}=y \mid Y^{n}, W=i\right) \\
& =\sum_{x \in \mathcal{X}} \mathbb{P}\left(X_{n+1}=x \mid W=i, Y^{n}\right) \mathbb{P}\left(Y_{n+1}=y \mid X_{n+1}=x, W=i, Y^{n}\right) \\
& =\sum_{x \in \mathcal{X}} \mathbb{P}\left(X_{n+1}=x \mid W=i, Y^{n}\right) \mathbb{P}\left(Y_{n+1}=y \mid X_{n+1}=x\right) \\
& =\sum_{x \in \mathcal{X}} \alpha_{i x} P_{Y \mid X}(y \mid x) .
\end{aligned}
$$


Here, (E.13) follows from the Markov chain $W-X_{n+1}-Y_{n+1}$ and (E.14) follows from the stationarity of the distribution $\mathbb{P}\left(Y_{n+1}=y \mid X_{n+1}=x\right)$ in $n$, which is derived from the stationarity of the distribution $\mathbb{P}\left(Y_{n+1}=y \mid X_{n+1}=x\right)$ in $n$. Similarly, we have

$$
\begin{aligned}
p(y \mid W \neq i) & =\mathbb{P}\left(Y_{n+1}=y \mid Y^{n}, W \neq i\right) \\
& =\sum_{x \in \mathcal{X}} \mathbb{P}\left(X_{n+1}=x \mid W \neq i, Y^{n}\right) \mathbb{P}\left(Y_{n+1}=y \mid X_{n+1}=x, W \neq i, Y^{n}\right) \\
& =\sum_{x \in \mathcal{X}} \mathbb{P}\left(X_{n+1}=x \mid W \neq i, Y^{n}\right) \mathbb{P}\left(Y_{n+1}=y \mid X_{n+1}=x\right) \\
& =\sum_{x \in \mathcal{X}} \beta_{i x} P_{Y \mid X}(y \mid x) .
\end{aligned}
$$

It is easy to see that for each fixed message $i \in \mathcal{W}=\{1, \ldots, M\}$ we have

$$
\sum_{x \in \mathcal{X}} \alpha_{i x}=\sum_{x \in \mathcal{X}} \beta_{i x}=1, \quad \alpha_{i x} \geq 0, \beta_{i x} \geq 0 .
$$

Observe that $F_{i}$ is a function of variables $p_{i},\left\{\alpha_{i x}\right\}$ and $\left\{\beta_{i x}\right\}$. For the purpose of finding an upper bound on $\max _{i}\left\{F_{i}\right\}$ in (E.7), we can consider only the constraints in (E.19) and find the maximization of $F_{i}$ over this convex set since other constraints that define the feasible set will only make $F_{i}$ smaller. With this consideration, let us consider find the maximization of $F_{i}$ over $\left\{\beta_{i x}\right\}$ with the assumption that $\sum_{x \in \mathcal{X}} \beta_{i x}=1$ and $\beta_{i x} \geq 0$. Fix an arbitrary $x^{\prime} \in \mathcal{X}$, then we have $\beta_{i x^{\prime}}=$ $1-\sum_{x \in \mathcal{X} \backslash\left\{x^{\prime}\right\}} \beta_{i x}$. We readily obtain that the derivatives of $F_{i}$ for any $x \in \mathcal{X} \backslash\left\{x^{\prime}\right\}$ are

$$
\begin{aligned}
\frac{\mathrm{d}^{2} F_{i}}{\mathrm{~d} \beta_{i x}^{2}} & =\frac{\partial^{2} F_{i}}{\partial \beta_{i x}^{2}}+\frac{\partial^{2} F_{i}}{\partial \beta_{i x^{\prime}}^{2}}-2 \frac{\partial^{2} F_{i}}{\partial \beta_{i x} \partial \beta_{i x^{\prime}}}, \\
\frac{\partial^{2} F_{i}}{\partial \beta_{i x} \partial \beta_{i x^{\prime}}} & =\left(1-p_{i}\right)^{2} \sum_{y \in \mathcal{Y}} \frac{\partial^{2} F_{i}}{\partial p(y)^{2}} P_{Y \mid X}(y \mid x) P_{Y \mid X}\left(y \mid x^{\prime}\right), \\
\frac{\partial^{2} F_{i}}{\partial p(y)^{2}} & =\frac{1}{p(y)}\left[1-\left(\ln \frac{p(y)}{p_{i} p(y \mid W=i)}\right)^{-1}+\left(\ln \frac{p(y)}{p_{i} p(y \mid W=i)}\right)^{-2}\right]>0 .
\end{aligned}
$$

Hence, from (E.20) to (E.22) we obtain

$$
\frac{\mathrm{d}^{2} F_{i}}{\mathrm{~d} \beta_{i x}^{2}}=\left(1-p_{i}\right)^{2} \sum_{y \in \mathcal{Y}} \frac{\partial^{2} F_{i}}{\partial p(y)^{2}}\left(P_{Y \mid X}(y \mid x)-P_{Y \mid X}\left(y \mid x^{\prime}\right)\right)^{2} \geq 0,
$$

for any $x \in \mathcal{X} \backslash\left\{x^{\prime}\right\}$.

If for all $x \in \mathcal{X} \backslash\left\{x^{\prime}\right\}$ we have $D\left(P_{Y \mid X}(\cdot \mid x) \| P_{Y \mid X}\left(\cdot \mid x^{\prime}\right)\right)=0$, it follows that

$$
\begin{aligned}
p(y \mid W=i) & =\sum_{x \in \mathcal{X}} \alpha_{i x} P_{Y \mid X}(y \mid x) \\
& =\sum_{x \in \mathcal{X}} \alpha_{i x} P_{Y \mid X}\left(y \mid x^{\prime}\right) \\
& =\sum_{x \in \mathcal{X} \backslash\left\{x^{\prime}\right\}} \alpha_{i x} P_{Y \mid X}\left(y \mid x^{\prime}\right)+\alpha_{i x^{\prime}} P_{Y \mid X}\left(y \mid x^{\prime}\right) \\
& =\left(1-\alpha_{i x^{\prime}}\right) P_{Y \mid X}\left(y \mid x^{\prime}\right)+\alpha_{i x^{\prime}} P_{Y \mid X}\left(y \mid x^{\prime}\right) \\
& =\left(1-\alpha_{i x^{\prime}}\right) P_{Y \mid X}(y \mid x)+\alpha_{i x^{\prime}} P_{Y \mid X}(y \mid x) \\
& =P_{Y \mid X}(y \mid x),
\end{aligned}
$$

for any $i \in \mathcal{W}$ and $y \in \mathcal{Y}$. In combination with the fact that the message is uniformly distributed on the message set $\mathcal{W}$, we obtain

$$
p(y \mid W \neq i)=P_{Y \mid X}(y \mid x) .
$$

Hence, it is easy to show that

$$
p(y)=P_{Y \mid X}(y \mid x), \quad \text { and } \quad p_{i}(y)=p_{i},
$$

for all $i \in \mathcal{W}$ and $y \in \mathcal{Y}$. Therefore, we have

$$
\mathbb{E}\left[\ln \mathcal{H}\left(W \mid Y^{n}\right)-\ln \mathcal{H}\left(W \mid Y^{n+1}\right) \mid Y^{n}\right]=0 .
$$


Now, we treat the remaining case where the relative entropy is positive. For any $x \in \mathcal{X}$ there always exists an $x^{\prime} \in \mathcal{X} \backslash\{x\}$ such that $D\left(P_{Y \mid X}(\cdot \mid x) \| P_{Y \mid X}\left(\cdot \mid x^{\prime}\right)\right)>0$. By choosing $x^{\prime}$ as a fixed symbol satisfying $D\left(P_{Y \mid X}(\cdot \mid x) \| P_{Y \mid X}\left(\cdot \mid x^{\prime}\right)\right)>0$, (E.23) becomes a strict inequality. Therefore, $\beta_{i x}$ must be zero or one. Consequently, for all fixed $i \in \mathcal{W}$, all the values of $\beta_{i x}$ for all $x \in \mathcal{X}$ except for one are zero.

Similarly, for any $x \in \mathcal{X} \backslash\left\{x^{\prime}\right\}$ such that $D\left(P_{Y \mid X}(\cdot \mid x) \| P_{Y \mid X}\left(\cdot \mid x^{\prime}\right)\right)>0$, we have

$$
\begin{aligned}
\frac{\partial^{2} F_{i}}{\partial \alpha_{i x}^{2}}=\sum_{y \in \mathcal{Y}}\left(P_{Y \mid X}(y \mid x)-P_{Y \mid X}\left(y \mid x^{\prime}\right)\right)^{2} & \frac{\left[p(y)-p_{i} p(y \mid W=i)\right]^{2}}{p(y) p^{2}(y \mid W=i)} \\
& \times\left[1-\left(\ln \frac{p(y)}{p_{i} p(y \mid W=i)}\right)^{-1}+\left(\ln \frac{p(y)}{p_{i} p(y \mid W=i)}\right)^{-2}\right]>0 .
\end{aligned}
$$

Consequently, either $\alpha_{i x}=0$ or $\alpha_{i x}=1, x \in \mathcal{X}$.

From (E.7), (E.9), and (E.10) together with above results, we obtain

$$
\mathbb{E}\left[\mathcal{H}\left(W \mid Y^{n}\right)-\mathcal{H}\left(W \mid Y^{n+1}\right) \mid Y^{n}\right] \leq \max \left\{0, \max _{x, x^{\prime}} \max _{\eta}\left\{\sum_{y \in \mathcal{Y}} p(y) \ln \frac{\eta \ln \eta}{f(y) \ln f(y)}\right\}\right\}
$$

where $\eta \in\left\{p_{1}, p_{2}, \ldots, p_{M}\right\},\left(x, x^{\prime}\right) \in \mathcal{X}^{2}$, and

$$
\begin{aligned}
& p(y)=\eta P_{Y \mid X}(y \mid x)+(1-\eta) P_{Y \mid X}\left(y \mid x^{\prime}\right), \\
& f(y)=\eta \frac{P_{Y \mid X}(y \mid x)}{p(y)} .
\end{aligned}
$$

We see from (E.35) and (E.36) that

$$
\sum_{y \in \mathcal{Y}} p(y) \ln \frac{\eta \ln \eta}{f(y) \ln f(y)}=\sum_{y \in \mathcal{Y}} p(y) \ln \left[\frac{p^{2}(y)}{P_{Y \mid X}(y \mid x) P_{Y \mid X}\left(y \mid x^{\prime}\right)}\right]+\sum_{y \in \mathcal{Y}} p(y) \ln \left[\frac{P_{Y \mid X}\left(y \mid x^{\prime}\right) \ln \eta}{p(y) \ln f(y)}\right] .
$$

Note that

$$
\frac{P_{Y \mid X}\left(y \mid x^{\prime}\right)}{p(y)}=\frac{1-f(y)}{1-\eta}
$$

It follows that

$$
\begin{aligned}
\ln \left[\frac{P_{Y \mid X}\left(y \mid x^{\prime}\right) \ln \eta}{p(y) \ln f(y)}\right] & =\ln \left[\frac{(1-f(y)) \ln \eta}{(1-\eta) \ln f(y)}\right] \\
& =[\ln (1-f(y))-\ln (-\ln f(y))]-[\ln (1-\eta)-\ln (-\ln \eta)] .
\end{aligned}
$$

From (E.36), we have

$$
\sum_{y \in \mathcal{Y}} p(y) f(y)=\sum_{y \in \mathcal{Y}} \eta P_{Y \mid X}(y \mid x)=\eta .
$$

Combining with the fact that $t \mapsto \ln (1-t)-\ln (-\ln t)$ is concave on $(0,1)[9, \mathrm{pp} .424]$, we obtain the following almost surely

$$
\sum_{y \in \mathcal{Y}} p(y)[\ln (1-f(y))-\ln (-\ln f(y))] \leq \ln (1-\eta)-\ln (-\ln \eta) .
$$

Note that $p(y)$ and $\eta$ are random because they depend on $Y^{n}=\left(Y_{1}, \ldots, Y_{n}\right)$ which is random (cf. (E.1) and (E.2)). The inequality in (E.42) means that

$$
\sum_{y \in \mathcal{Y}} p(y) \ln \left[\frac{P_{Y \mid X}\left(y \mid x^{\prime}\right) \ln \eta}{p(y) \ln f(y)}\right] \leq 0 .
$$

In addition, by Jensen's inequality and the definition of $p(y)$ in (E.35) (see the first inequality in Eqn. (2.6) of [9] for an analogous derivation), it holds that

$$
\begin{aligned}
& p(y) \ln \left[\frac{p^{2}(y)}{P_{Y \mid X}(y \mid x) P_{Y \mid X}\left(y \mid x^{\prime}\right)}\right] \\
& \leq \eta P_{Y \mid X}(y \mid x) \ln \frac{P_{Y \mid X}(y \mid x)}{P_{Y \mid X}\left(y \mid x^{\prime}\right)}+(1-\eta) P_{Y \mid X}\left(y \mid x^{\prime}\right) \ln \frac{P_{Y \mid X}\left(y \mid x^{\prime}\right)}{P_{Y \mid X}(y \mid x)}
\end{aligned}
$$


Hence, we obtain

$$
\begin{aligned}
& \sum_{y \in \mathcal{Y}} p(y) \ln \left[\frac{p^{2}(y)}{P_{Y \mid X}(y \mid x) P_{Y \mid X}\left(y \mid x^{\prime}\right)}\right] \\
& \leq \eta D\left(P_{Y \mid X}(\cdot \mid x) \| P_{Y \mid X}\left(\cdot \mid x^{\prime}\right)\right)+(1-\eta) D\left(P_{Y \mid X}\left(\cdot \mid x^{\prime}\right) \| P_{Y \mid X}(\cdot \mid x)\right) \\
& \leq B \quad \text { a.s. }
\end{aligned}
$$

From (E.34), (E.37), (E.43), and (E.46) we obtain (110), concluding the proof of Lemma 7.

\section{APPENDIX F}

\section{PROOF OF LEMMA 9}

From (113) and definition of the limit inferior [31, Definition 3.16], there exists a subsequence $\left\{x_{n_{k}}\right\}_{k=1}^{\infty}$ such that $\lim _{k \rightarrow \infty} x_{n_{k}}=0$. It then follows from (112) that $\limsup _{k \rightarrow \infty} y_{n_{k}}=0$. In addition, since $\left\{y_{n}\right\}_{n \in \mathbb{R}_{+}}$is non-negative, $\lim _{k \rightarrow \infty} y_{n_{k}}=0$. Since $\left\{y_{n}\right\}_{n \in \mathbb{R}_{+}}$is non-negative, again from the definition of the limit inferior, (114) holds.

\section{APPENDIX G}

\section{PROOF OF LEMMA 10}

Assume, to the contrary of (116), that

$$
\limsup _{N \rightarrow \infty}-\frac{\ln \phi_{N}}{N}=+\infty .
$$

By definition of the limit superior [31, Definition 3.16], there exists an increasing sequence of indices $\left\{N_{k}\right\}_{k=1}^{\infty}$ such that

$$
\lim _{k \rightarrow \infty}-\frac{\ln \phi_{N_{k}}}{N_{k}}=+\infty
$$

Then, we have

$$
\begin{aligned}
& \limsup _{k \rightarrow \infty}\left[-\frac{\ln \phi_{N_{k}}}{N_{k} \rho_{N_{k}}}-\frac{\ln \left(N_{k}\left(C-\rho_{N_{k}}\right)-\ln \phi_{N_{k}}\right)}{N_{k} \rho_{N_{k}}}\right] \\
& =\limsup _{k \rightarrow \infty}-\frac{\ln \phi_{N_{k}}}{N_{k}}\left[\frac{1}{\rho_{N_{k}}}-\frac{\ln \left(\frac{C N_{k}-N_{k} \rho_{N_{k}}}{N_{k}}-\frac{\ln \phi_{N_{k}}}{N_{k}}\right)+\ln N_{k}}{-\frac{\ln \phi_{N_{k}}}{N_{k}}} \cdot \frac{1}{\sqrt{N_{k}}\left(\sqrt{N_{k}} \rho_{N_{k}}\right)}\right] \\
& =\lim _{k \rightarrow \infty}-\frac{\ln \phi_{N_{k}}}{N_{k}}\left[\frac{1}{\rho_{N_{k}}}-\frac{\ln \left(\frac{C N_{k}-N_{k} \rho_{N_{k}}}{N_{k}}-\frac{\ln \phi_{N_{k}}}{N_{k}}\right)}{-\frac{\ln \phi_{N_{k}}}{N_{k}}} \cdot \frac{1}{\sqrt{N_{k}}\left(\sqrt{N_{k}} \rho_{N_{k}}\right)}\right]-\frac{\ln N_{k}}{\sqrt{N_{k}}} \cdot \frac{1}{\sqrt{N_{k}} \rho_{N_{k}}} \\
& =+\infty .
\end{aligned}
$$

On the other hand, from (G.1) we have

$$
-\frac{\ln \phi_{N_{k}}}{N_{k}} \geq 1
$$

for $k$ sufficiently large. This is equivalent to

$$
0 \leq \phi_{N_{k}} \leq \exp \left(-N_{k}\right)
$$

for $k$ sufficiently large. Hence, we obtain

$$
0 \leq \limsup _{k \rightarrow \infty} \frac{\phi_{N_{k}}}{\rho_{N_{k}}} \leq \limsup _{k \rightarrow \infty} \frac{\exp \left(-N_{k}\right)}{\rho_{N_{k}}}=\limsup _{k \rightarrow \infty}\left[\exp \left(-N_{k}\right) \sqrt{N_{k}}\right]\left[\frac{1}{\sqrt{N_{k}} \rho_{N_{k}}}\right]=0
$$

i.e., $\lim _{k \rightarrow \infty} \phi_{N_{k}} / \rho_{N_{k}}=0$. It follows that

$$
\begin{aligned}
& \limsup _{k \rightarrow \infty}\left[\frac{B}{C N_{k} \rho_{N_{k}}}+\frac{B \phi_{N_{k}}}{\rho_{N_{k}}}-\frac{B \phi_{N_{k}}}{C}+\frac{B}{C}+\frac{O(1)}{N_{k} \rho_{N_{k}}}\right] \\
& \quad \leq \limsup _{k \rightarrow \infty} \frac{B}{C N_{k} \rho_{N_{k}}}+\limsup _{k \rightarrow \infty} \frac{B \phi_{N_{k}}}{\rho_{N_{k}}}-\liminf _{k \rightarrow \infty} \frac{B \phi_{N_{k}}}{C}+\frac{B}{C}+\limsup _{k \rightarrow \infty} \frac{O(1)}{N_{k} \rho_{N_{k}}}=\frac{B}{C} .
\end{aligned}
$$

On the other hand, from (115) we have

$$
\begin{aligned}
& -\frac{\ln \phi_{N_{k}}}{N_{k} \rho_{N_{k}}}-\frac{\ln \left(N_{k}\left(C-\rho_{N, k}\right)-\ln \phi_{N_{k}}\right)}{N_{k} \rho_{N_{k}}} \\
& \quad \leq \frac{B}{C N_{k} \rho_{N_{k}}}+\frac{B \phi_{N_{k}}}{\rho_{N_{k}}}-\frac{B \phi_{N_{k}}}{C}+\frac{B}{C}+\frac{O(1)}{N_{k} \rho_{N_{k}}} .
\end{aligned}
$$


From (G.4), (G.6), (G.8), and (G.9) we obtain

$$
\begin{aligned}
+\infty & =\limsup _{k \rightarrow \infty}\left[-\frac{\ln \phi_{N_{k}}}{N_{k} \rho_{N_{k}}}-\frac{\ln \left(N_{k}\left(C-\rho_{N, k}\right)-\ln \phi_{N_{k}}\right)}{N_{k} \rho_{N_{k}}}\right] \\
& \leq \limsup _{k \rightarrow \infty}\left[\frac{B}{C N_{k} \rho_{N_{k}}}+\frac{B \phi_{N_{k}}}{\rho_{N_{k}}}-\frac{B \phi_{N_{k}}}{C}+\frac{B}{C}+\frac{O(1)}{N_{k} \rho_{N_{k}}}\right] \leq \frac{B}{C} .
\end{aligned}
$$

This is a contradiction, concluding the proof of Lemma 9.

Acknowledgements: The authors would like to sincerely thank Dr. Mladen Kovačević (National University of Singapore) for useful comments that helped to greatly improve the manuscript.

\section{REFERENCES}

[1] C. E. Shannon. The zero error capacity of a noisy channel. IRE Trans. on Inform. Th., 2(3):8-19, 1956.

[2] J. Schalkwijk and T. Kailath. A coding scheme for additive noise channels with feedback-Part I: No bandwith constraint. IEEE Trans. on Inform. Th., 12(2):172-182, 1966.

[3] O. Shayevitz and M. Feder. Optimal feedback communication via posterior matching. IEEE Trans. on Inform. Th., 57(3):1186-1222, 2011.

[4] M. V. Burnashev. Data transmission over a discrete channel with feedback. Random transmission time. Problemy Peredachi Informatsii (Problems of Information Transmission), 12(4):10-30, 1976.

[5] Y. Polyanskiy, H. V. Poor, and S. Verdú. Feedback in the non-asymptotic regime. IEEE Trans. on Inform. Th., 57(8):4903-4925, 2011.

[6] Y. Altuğ and A. B. Wagner. Moderate deviations in channel coding. IEEE Trans. on Inform. Th., 60(8):4417-4426, 2014.

[7] Y. Polyanskiy and S. Verdú. Channel dispersion and moderate deviations limits for memoryless channels. In Proc. of Allerton Conference, pages 1334-1339, Monticello, IL, 2010.

[8] H. Yamamoto and K. Itoh. Asymptotic performance of a modified Schalkwijk-Barron scheme for channels with noiseless feedback. IEEE Trans. on Inform. Th., 25(6):729-733, 1979.

[9] M. V. Burnashev. Sequential discrimination of hypotheses with control of observations. Math. USSR Izv. (in Russian), 15(3):419-440, 1980.

[10] P. Berlin, B. Nakiboğlu, B. Rimoldi, and E. Telatar. A simple converse of Burnashev's reliability function. IEEE Trans. on Inform. Th., 55(7):3074-3080, 2009.

[11] B. Nakiboğlu and R. G. Gallager. Error exponents for variable-length block codes with feedback and cost constraints. IEEE Trans. on Inform. Th., 54(3):945-963, 2008.

[12] A. Mahajan and S. Tatikonda. Opportunistic capacity and error exponent region for the compound channel with feedback. IEEE Trans. on Inform. Th., 58(7):4331-4341, 2012.

[13] D. Blackwell, L. Breiman, and A. Thomasian. The capacity of a class of channels. Ann. Math. Statist., 30(4):1229-1241, 1959.

[14] A. Tchamkerten and I. E. Telatar. On the universality of Burnashev's exponent. IEEE Trans. on Inform. Th., 51(8):2940-2944, 2005.

[15] A. Tchamkerten and I. E. Telatar. On the use of training sequences for channel estimation. IEEE Trans. on Inform. Th., 52(3):1171-1176, 2006.

[16] A. Tchamkerten and I. E. Telatar. Variable length coding over an unknown channel. IEEE Trans. on Inform. Th., 52(5):2126-2145, 2006.

[17] V. Kostina, Y. Polyanskiy, and S. Verdú. Joint source-channel coding with feedback. IEEE Trans. on Inform. Th., 63(6):3502-3515, 2017.

[18] J. Chen, D.-K. He, A. Jagmohan, and L. A. Lastras-Montano. On the redundancy-error tradeoff in Slepian-Wolf coding and channel coding. In ISIT, pages 1326-1330, Nice, France, 2007.

[19] D. K. He, L. A. Lastras-Montano, E. H. Yang, A. Jagmohan, and J. Chen. On the redundancy of Slepian-Wolf coding. IEEE Trans. on Inform. Th., 55(12):5607-5627, 2009

[20] V. Y. F. Tan. Moderate deviations of lossy source coding for discrete and Gaussian sources. In Proc. of Intl. Symp. on Inform. Th., pages 920-924, Cambridge, MA, 2012

[21] Y. Altuğ, A. B. Wagner, and I. Kontoyiannis. Lossless compression with moderate error probability. In Proc. of Intl. Symp. on Inform. Th., pages 1744-1748, Istanbul, Turkey, 2013.

[22] Y. Altuğ, H. V. Poor, and S. Verdú. On fixed-length channel coding with feedback in the moderate deviations regime. In Proc. of Intl. Symp. on Inform. Th., pages 1816-1820, Hong Kong, China, 2015.

[23] M. Hayashi and V. Y. F. Tan. Asymmetric evaluations of erasure and undetected error probabilities. IEEE Trans. on Inform. Th., 61(12):6560-6577, 2015.

[24] S.-H. Lee, V. Y. F. Tan, and A. Khisti. Streaming data transmission in the moderate deviations and central limit regimes. IEEE Trans. on Inform. Th., 62(12):6816-6830, 2016.

[25] P. Billingsley. Probability and Measure. Wiley-Interscience, 3rd edition, 1995.

[26] M. V. Burnashev and K. Sh. Zigangirov. On one problem in observation control. Math. USSR Izv. (in Russian), 11(3):44-52, 1975.

[27] M. Naghshvar, T. Javidi, and M. Wigger. Extrinsic Jensen-Shannon divergence: Applications to variable-length coding. IEEE Trans. on Inform. Th., 61(4):2148-2164, 2015.

[28] R. G. Gallager. Information Theory and Reliable Communication. Wiley, New York, 1968.

[29] P. Hall and C. C. Heyde. Martingale Limit Theory and Its Application. Academic Press, 1991.

[30] H. Royden and P. Fitzpatrick. Real Analysis. Pearson, 4th edition, 2010.

[31] W. Rudin. Principles of Mathematical Analysis. McGraw-Hill, 1976. 